$P M$

PMCA

PSD

SERCA Sarco/Endoplasmic Reticulum $\mathrm{Ca}^{2+}$-ATPase

SpApp Spine Apparatus

STDP Spike-Timing Dependent Plasticity

VSCC Voltage Sensitive Calcium Channel

\section{Keywords:}

\section{Abbreviations:}

BPAP Back Propagating Action Potential

EPSP Excitatory Postsynaptic Potential

LTD Long Term Depression

LTP Long Term Potentiation

NCX Sodium-Calcium Exchanger

NMDAR N-methyl-D-aspartate Receptor

Plasma Membrane

Plasma Membrane $\mathrm{Ca}^{2+}$-ATPase

\title{
Stochastic simulations reveal that dendritic spine morphology regulates synaptic plasticity in a deterministic manner
}

\author{
M. V. Holst*, M. K. Bell*, C. T. Lee, and P. Rangamani** \\ Department of Mechanical and Aerospace Engineering, \\ University of California San Diego, La Jolla CA 92093. \\ *These authors contributed equally \\ **To whom correspondence must be addressed: prangamani@ucsd.edu
}

\begin{abstract}
Dendritic spines act as computational units and must adapt their responses according to their activation history. Calcium influx acts as the first signaling step during postsynaptic activation and is a determinant of synaptic weight change. Dendritic spines also come in a variety of sizes and shapes. To probe the relationship between calcium dynamics and spine morphology, we used a stochastic reaction-diffusion model of calcium dynamics in idealized and realistic geometries. We show that despite the stochastic nature of the various calcium channels, receptors, and pumps, spine size and shape can separately modulate calcium dynamics and subsequently synaptic weight updates in a deterministic manner. The relationships between calcium dynamics and spine morphology identified in idealized geometries also hold in realistic geometries suggesting that there are geometrically determined deterministic relationships that may modulate synaptic weight change.
\end{abstract}

Calcium | dendritic spine | morphology | synaptic weight | spine apparatus

AMPAR $\quad \alpha$-amino-3-hydroxy-5-methyl-4-isoxazolepropionic Acid Receptor 


\section{Introduction}

Dendritic spines are small protrusions along the dendrites of neurons that compartmentalize postsynaptic biochemical, electrical, and mechanical responses. These subcompartments house the majority of excitatory synapses and are key for neuronal communication and function (1, 2). Because of their unique biochemical compartmentation capabilities, spines are thought of as computational units that can modify their synaptic strength through a process called synaptic plasticity (1) 3).

Calcium plays a key role as a second messenger in biochemical and physical modifications during synaptic plasticity, triggering downstream signaling cascades within dendritic spines, and the entire neuron (3-5). Efforts have also linked calcium levels to synaptic weight change (6-9). Synaptic weight update refers to the change in the strength of the postsynaptic response in the event of neurotransmitter release from the presynapse. Calcium levels have often been used as an indicator of the early events preceding the complex downstream signaling (7, 10, 12), specifically the modulation of $\alpha$-amino-3-hydroxy-5-methyl-4-isoxazolepropionic Acid Receptor (AMPAR) density (13), and thus inform the synaptic weight update. An increase in synaptic weight is associated with Long Term Potentiation (LTP), while a decrease in synaptic weight is associated with Long Term Depression (LTD) (14, 15). While synaptic weight update requires a host of downstream signaling and mechanical interactions, the level of calcium can be thought of as an indicator of synaptic plasticity and weight $(7,16)$.

Dendritic spines have characteristic sizes and shapes that dynamically change over time in response to stimulus, and are associated with their function and synaptic plasticity (17). Just as whole cell shape is known to influence signaling dynamics (18-21), studies have specifically probed the interplay between calcium dynamics and dendritic spine morphology (4, 22, 24). Due to the historical significance of dendritic spines as electrical subcompartments, the morphology of the spine neck has been implicated in regulating calcium signaling and longer spine necks were found to decouple spine-dendrite calcium signaling (25). Additional modeling work coupled actinmyosin contractions to cytoplasmic flow to identify two timescales of calcium motion, driven by flow and diffusion respectively, that depend on spine geometry (26). A combined analytical and numerical study showed how geometry and curvature gives rise to pseudo-harmonic functions that can predict the locations of maximum and minimum calcium concentration (23). More recently, we used a deterministic reaction-diffusion model to investigate dendritic spine morphology and ultrastructure, and found that dendritic spine volume-to-surface area ratios and the presence of spine apparatus modulate calcium levels (22).

Due the small volume of dendritic spines, stochastic calculations are important to gain insight into the spatiotemporal dynamics of spine calcium; there are approximately seven ions of calcium in a resting spine (24,27). Due to their probabilistic nature and discrete number, calcium channels and receptors appear to behave stochastically (28-30). This indicates that the system leans towards stochasticity and it has been suggested that synaptic plasticity itself relies on stochasticity for robustness (28, 31, 32). In this work, using idealized and realistic spine geometries, we investigate the impact of shape and stochasticity on calcium dynamics and synaptic weight change. We seek to answer the following specific question: How do specific geometric parameters - namely shape and size of dendritic spines - influence calcium dynamics? To address these questions, we built a spatial, stochastic model of calcium dynamics in various dendritic spines geometries. We used idealized geometries to control for various geometric parameters and then extended our calculations to realistic geometries. We probed the influence of spine shape, volume, and volume-to-surface area ratio on calcium influx, variance of calcium dynamics, and the robustness of synaptic weight. We show that although calcium dynamics in individual spines is stochastic, the key readouts from 


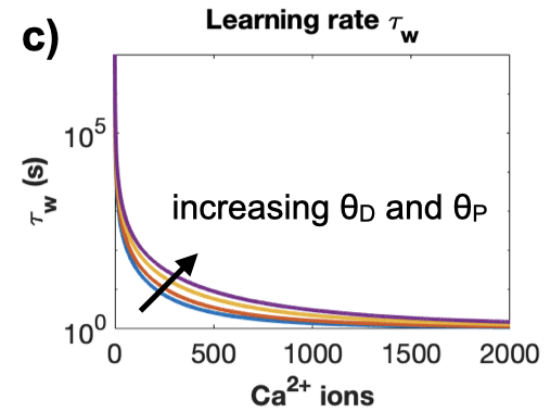

the model, including mean calcium and synaptic weight update, behave deterministically with respect to the variation of geometric parameters.
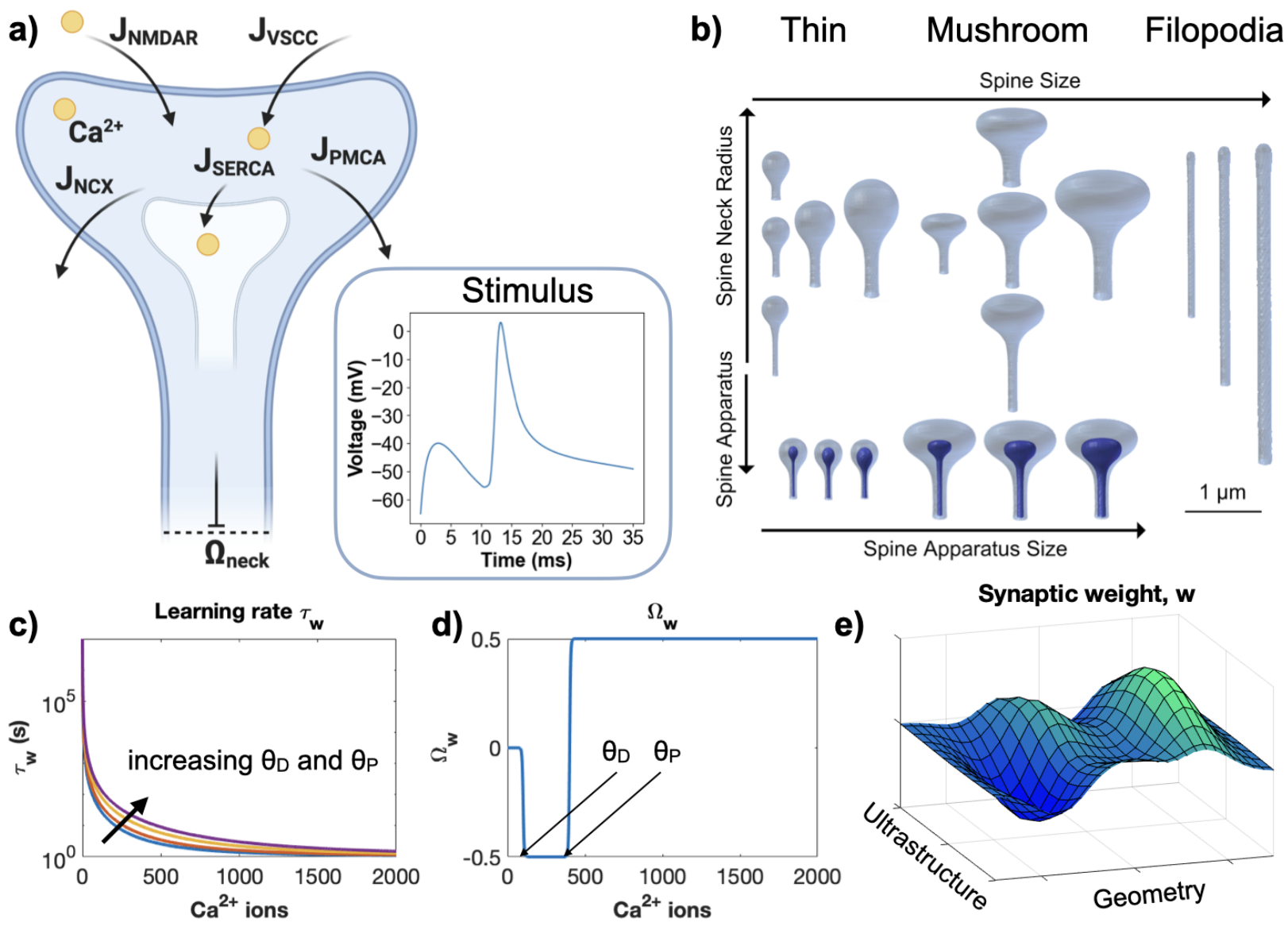

Figure 1: Model overview. a) Our stochastic model includes calcium influx through NMDAR and VSCC calcium efflux to the extracellular space through PMCA and NCX pumps, and to the Spine Apparatus through SERCA pumps. Arrows indicate the movement of $\mathrm{Ca}^{2+}$ through the labeled pump, channel, or receptor. $\Omega_{\text {neck }}$ represents the Dirichlet boundary condition at the base of the spine neck, at which the concentration of calcium ions is clamped to zero. Cytosolic calcium is buffered using mobile and immobile calcium buffers. Inset: A change in membrane potential triggered by an excitatory postsynaptic action potential (EPSP) and back propagating action potential (BPAP) acts as the model stimulus. b) The geometric factors considered in our model include spine shape, spine size, neck radius and length, and SpApp size. We investigate three spine shapes: thin, mushroom, and filopodia-shaped. Calcium levels determine the learning rate $\tau_{w}$, (c), and function $\Omega_{w}$, (d), that in turn determine synaptic weight, (e). The influence of geometry and ultrastructure on calcium signaling thus has an influence on synaptic weight. $\theta_{D}$ and $\theta_{P}$ represent the thresholds for long term depression and potentiation, respectively. Panel a) was generated using biorender.com

\section{Results}

In this work, we sought to decipher the contributions of spine size and shape to synaptic weight change. We briefly summarize our model development strategy here as shown in Figure 1 . We conducted stochastic simulations of calcium influx through N-methyl-D-aspartate Receptor (NMDAR) and Voltage Sensitive Calcium Channels (VSCCs) based on (24). The system stimulus is a Excitatory Postsynaptic Potential (EPSP)] and Back Propagating Action Potential (BPAP)] offset by $10 \mathrm{~ms}(24)$. Calcium ions leave the spine volume through the pumps on the plasma membrane, Plasma Membrane $\mathrm{Ca}^{2+}$-ATPase (PMCA) and Sodium-Calcium Exchanger (NCX). 
and into the Spine Apparatus (SpApp) (if present) through Sarco/Endoplasmic Reticulum $\mathrm{Ca}^{2+}$ ATPase (SERCA), In addition, the base of the spine neck has a Dirichlet boundary condition of calcium clamped to zero. Mobile and immobile buffers are present in the cytoplasm to reversibly bind calcium, and there is an additional exponential decay throughout the cytoplasm. All simulations were performed using MCell (33-35) to capture the stochastic nature of calcium dynamics in the small spine volumes and each simulation condition was run with 50 random seeds. System configuration and analysis scripts are all available on Github https://github. com/RangamaniLabUCSD/StochasticSpineSimulations. Synaptic weight was calculated using an ordinary differential equation dependent on the total number of calcium ions in the cytoplasm at each time point, see Table 2 and Section 4.5. The rate of synaptic weight update depends on a learning rate, $\tau_{w}$, and a thresholding function, $\Omega_{w}$, that are both dependent on calcium ion levels, Figure $1 \mathrm{k}$-d. We investigate how spine geometry and ultrastructure can influence synaptic weight change (Figure 1 $1 \mathrm{k}$ ). Model geometries were selected as follows: idealized geometries of thin, mushroom, and filopodia-shaped geometries from Alimohamadi et al. (36), see Supp. Table 3 and 4. For each geometry, the Postsynaptic Density (PSD) area was set as a fixed proportion of the spine volume. We first investigate whether spine size has any effect on filopodia-shaped spines (Figure 2), thin spines (Figure 3), and mushroom spines (Figure 4). Next we consider the role of spine apparatus (Figure 5). Last we test the trends we find in idealized spines on realistic spine geometries (Figure 6). Our results predict that synaptic weight change through calcium dynamics is a deterministic function of geometric parameters of the spines (Figure 7). We note that our goal is not to provide a function fit but to demonstrate trends. We discuss these results in detail below.

a)

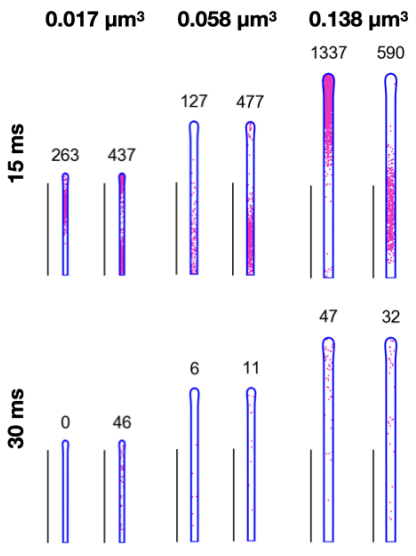

b)

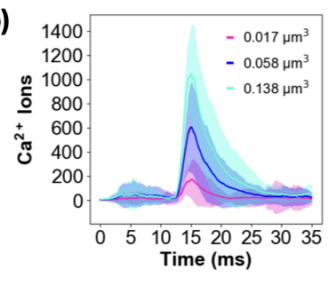

e)

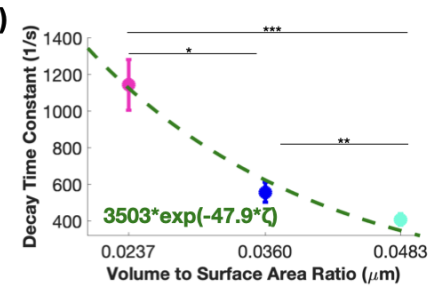

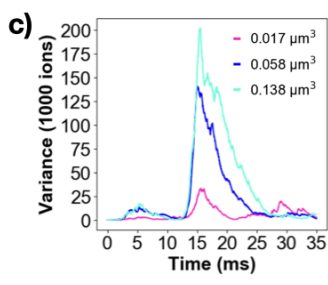

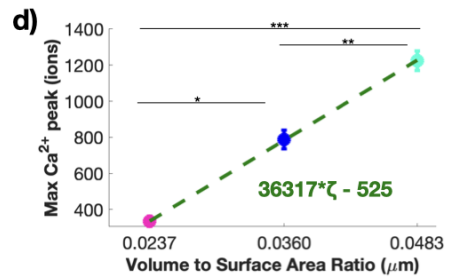

f)

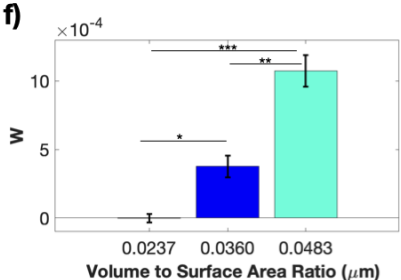

Figure 2: Calcium dynamics and synaptic weight change in filopodia-shaped spines depend on spine size. a) Spatial plots illustrating $\mathrm{Ca}^{2+}$ localization at 15 and $30 \mathrm{~ms}$ for filopodia-shaped spines with different volumes (0.017, 0.058 and $0.138 \mu^{3}$ ). The number above each geometry corresponds to the number of $\mathrm{Ca}^{2+}$ in that frame. Scale bars: $2 \mu \mathrm{m}$. b) Mean (solid) and standard deviation (shaded area) of $\mathrm{Ca}^{2+}$ transients across 50 simulations for each of the three filopodia-shaped spine sizes. c) Variance of $\mathrm{Ca}^{2+}$ over time. d) The mean and standard error $(n=50)$ of the peak number of $\mathrm{Ca}^{2+}$ in different filopodia-shaped spine sizes shows statistically significant differences; $\mathrm{p}^{*}=2.0262 \times 10^{-11}$; $\mathrm{p}^{\star *}=9.898 \times 10^{-8} ; \mathrm{p}^{* \star *}=4.362 \times 10^{-26}$ using a two-tailed $t$-test. We fit the trend in peak $\mathrm{Ca}^{2+}$ as a linear function of volume-to-surface area ratio, $\zeta ; r^{2}=0.5521$ for the linear fit. e) The decay timescales of each $\mathrm{Ca}^{2+}$ transient are estimated by fitting with an exponential decay function $c \cdot \exp (-k t)$. The mean and standard error $(n=50)$ of the decay time constant, $k$, shows statistically significant differences across filopodia-shaped spine sizes; $\mathrm{p}^{*}=1.6331 \times 10^{-4} ; \mathrm{p}^{* *}=$ $0.0209 ; \mathrm{p}^{\star * *}=1.3381 \times 10^{-6}$ from a two-tailed $t$-test. The mean decay time constants as a function of volume-to-surface area ratio, $\zeta$, was fit with an exponential $a \cdot \exp (-b \zeta) ; r^{2}=0.203$ for the exponential fit. f) The mean and standard error $(n=50)$ of the calculated synaptic weight change at the last time point in the simulation for all filopodia-shaped spine sizes, plotted against the volume-to-surface area ratio, shows statistically significant differences between all cases; $p^{*}$ $=2.7290 \times 10^{-5} ; \mathrm{p}^{\star *}=2.8626 \times 10^{-6} ; \mathrm{p}^{\star \star *}=1.6321 \times 10^{-14}$ from two-tailed $t$-test. 


\subsection{Synaptic weight change depends on spine volume-to-surface ratio in filopodia- shaped spines}

We begin our analysis with a simple question - does spine size alter synaptic weight change? To answer this question, we first examined filopodia-shaped spines. Dendritic filopodia are precursors of dendritic spines and serve to bridge the gap between the dendrite and an axon that is passing by during synapse formation (37). These are highly motile elongated structures that resemble tubules (lengths of 2-20 $\mu \mathrm{m}$ and neck diameters smaller than $0.3 \mu \mathrm{m}$ ). The simplicity of this geometry allows us to focus on the role of size alone in a simple spine geometry. We used spine geometries of three different volumes $\left(0.017,0.058\right.$ and $\left.0.138 \mu \mathrm{m}^{3}\right)$. Simulations revealed that the calcium dynamics in these tubule-shaped spines appeared to follow a 'plug-flow' behavior where at $15 \mathrm{~ms}$, all the calcium is localized to one region (Figure 2a). This behavior is because of the narrow geometry of the spine, preventing dispersion of the calcium (see also Supplemental Movie S1). Next, we look at the temporal dynamics of calcium and note that the larger spines have larger numbers of calcium ions (Figure $2 \mathrm{~b}$ ) but also have a larger variance of calcium ions (Figure $2 \mathrm{~b}$ ). We further characterized the dynamics by considering the peak calcium values and decay time constants of the calcium transients versus the spine volume-to-surface area ratio. We chose the volume-to-surface area ratio as a geometric metric of spine morphology because it encompasses both the cytosolic volume through which calcium diffuses and the surface area of the spine membrane through which calcium can enter and leave the system. Additional analyses with respect to spine volume are shown in Figure S1.

We note that, indeed, increasing spine size and therefore the volume-to-surface ratio, causes a linearly proportional and significant increase in peak calcium ions (Figure $2 \mathrm{~d}$ ). We also found that the decay time of calcium from the peak decreased with increasing volume-to-surface area ratios and satisfied an exponential dependence (Figure 2k). As spine size increases, the decay time constant decreases, showing that it takes longer for calcium to clear out of the larger spines and spines with larger volume-to-surface area ratios. Finally, we calculated the synaptic weight change (see Supplemental Section 4.5) and compared this value at $35 \mathrm{~ms}$ across volume-to-surface area ratios for the filopodia-shaped spines (Figure 2f). We observed that while the smallest spine had no observable weight change presumably because of the net low calcium influx, the weight change increases with increase in spine volume-to-surface-area ratio (Figure 2 f). Thus, we find that even for a shape as simple as a filopodia-shaped spine, changes in spine volume-to-surface area ratio can dramatically alter calcium dynamics and synaptic weight change even in stochastic conditions suggesting a close coupling between spinogenesis and calcium handling.

\subsection{Thin and mushroom-shaped spines modulate synaptic weight changes as a function of volume-to-surface area ratio}

We next asked if the relationships of spine size and synaptic weight change observed for filopodiashaped spines (Figure 2) also holds for thin and mushroom-shaped spines. Thin and mushroomshaped spines emerge from filopodia-shaped spines as spinogenesis progresses (37, 38). While it has been proposed that spines exist in a continuum of shapes (39), historically it has been useful to categorize spines into specific categories of shapes (40). Thin spines, with small heads and thin necks, have been classified as 'write-enabled' or learning spines due to their high motility. Mushroom spines, on the other hand, with bulbous heads and relatively wider necks, are termed 'write-protected' or memory spines due to their stability (41). Thin spines are characterized by a spherical head and we repeated the calcium influx simulations in thin spines of three different volumes $\left(0.035,0.119\right.$ and $\left.0.283 \mu \mathrm{m}^{3}\right)$ that were informed by the ranges found in the literature, 


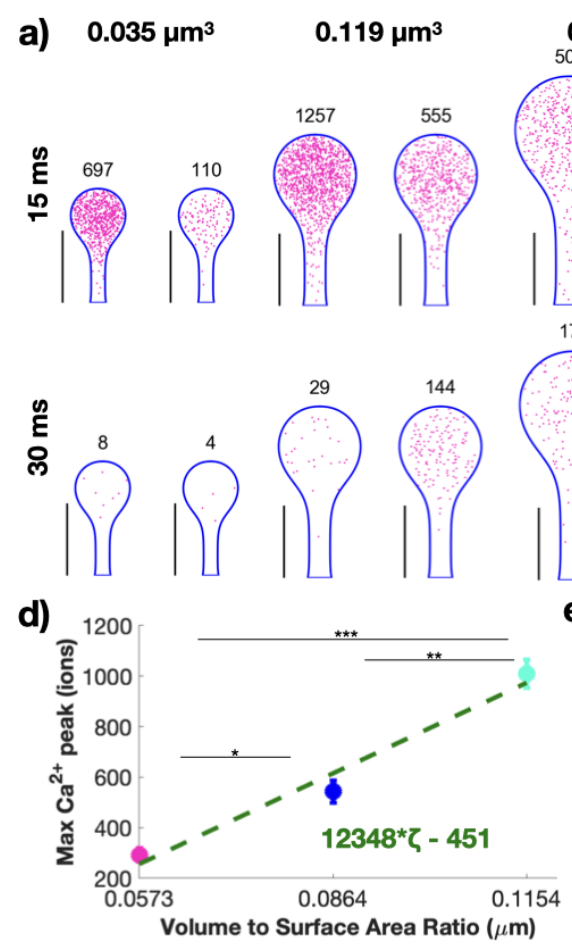

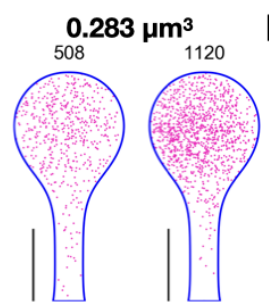

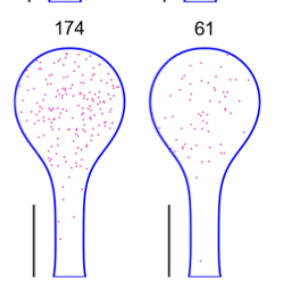

e)

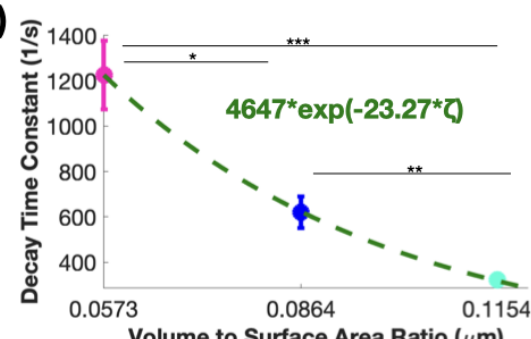

b)

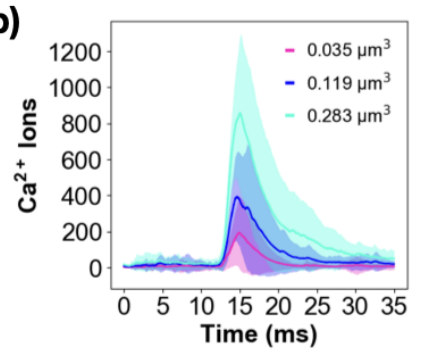

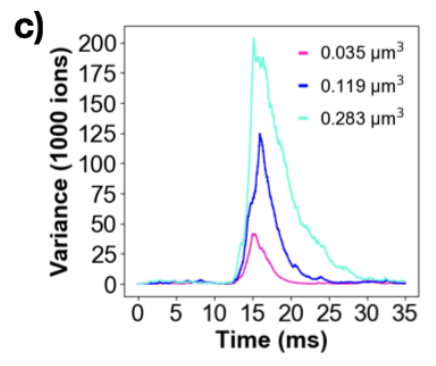

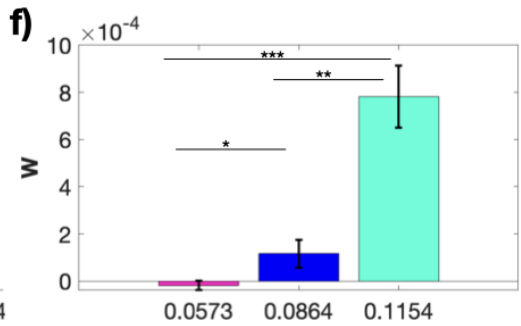

Volume to Surface Area Ratio $(\mu \mathrm{m})$

Figure 3: Changing thin spine size modulates calcium dynamics and synaptic weight change. a) Spatial plots illustrating $\mathrm{Ca}^{2+}$ localization at 15 and $30 \mathrm{~ms}$ for thin spines with different volumes $0.035,0.119$ and $0.283 \mu \mathrm{m}^{3}$ ). The number above each geometry corresponds to the number of $\mathrm{Ca}^{2+}$ in the frame. Scale bars: $0.5 \mu \mathrm{m}$. b) Mean (solid) and standard deviation (shaded area) of $\mathrm{Ca}^{2+}$ transients across 50 simulations for each of the three thin spine sizes. c) Variance of $\mathrm{Ca}^{2+}$ over time. d) The mean and standard error $(n=50)$ of the peak number of $\mathrm{Ca}^{2+}$ in different thin spine sizes shows statistically significant differences; $p^{*}=5.2641 \times 10^{-6} ; p^{* *}=2.7377 \times 10^{-9} ; p^{* * *}=5.0036 \times 10^{-20}$ from two-tailed $t$-test. We fit the trend in peak $\mathrm{Ca}^{2+}$ as a linear function of volume-to-surface area ratio, $\zeta ; r^{2}=0.4676$ for the linear fit. e) The decay timescales of each $\mathrm{Ca}^{2+}$ transient are estimated by fitting with an exponential decay function $c \cdot \exp (-k t)$. The mean and standard error $(n=50)$ of the decay time constant, $k$, shows statistically significant differences across thin spine sizes; $\mathrm{p}^{*}=4.3976 \times 10^{-4} ; \mathrm{p}^{\star \star}=1.1541 \times 10^{-4} ; \mathrm{p}^{\star \star *}=5.4590 \times 10^{-8}$ from two-tailed $t$-test. The mean decay time constants as a function of volume-to-surface area ratio, $\zeta$, was fit with an exponential $a \cdot \exp (-b \zeta)$; $r^{2}=0.2285$ for the exponential fit. f) The mean and standard error $(n=50)$ of the calculated synaptic weight change at the last time point in the simulation for all thin spine sizes, plotted against the volume-to-surface area ratio, shows statistically significant differences between all cases; $p^{\star}=0.0315 ; p^{\star *}=1.0661 \times 10^{-5} ; p^{\star \star *}=2.5751 \times 10^{-8}$ from twotailed $t$-test. 
Figure 3. We observe that, in thin spines, the calcium ions are concentrated in the head at $15 \mathrm{~ms}$ but disperse more uniformly by $30 \mathrm{~ms}$ (Figure 3 a and Supplemental Movie S2). We do not observe a plug-flow like behavior as we did for filopodia-shaped spines likely because of the differences in both shape and volume of the thin spines. Calcium dynamics in thin spines follows the expected temporal dynamics (Figure $3 \mathrm{~b}$ ), with larger spines having larger peak calcium and increased time to decay. Larger thin spines also have larger variance in the calcium ion concentration over time (Figure 3c). Next, we found that the maximum calcium ions per spine was significantly larger in larger spines with statistically different values for the different sized spines. The peak calcium increased linearly compared to spine volume-to-surface area but with a smaller slope when compared to the filopodia-shaped spines (max peak values in filopodia-shaped spines increased three times faster than those in thin spines), (Figure $3 \mathrm{~d}$ ). This suggests that the size dependence of calcium grows slower in thin spines than in filopodia-shaped spines. The decay time also showed an exponential decay in thin spines with increasing volume-to-surface area ratio (Figure 3 e). The exponent was smaller for thin spines when compared to filopodia-shaped spines (47.9 versus 23.27) suggesting that the decay rate with respect to volume-to-surface area ratio was slower in thin spines. Finally, the synaptic weight change showed an increase with volume-to-surface area ratio in thin spines (Figure 3f) indicating that larger spines are capable of stronger learning outcomes.

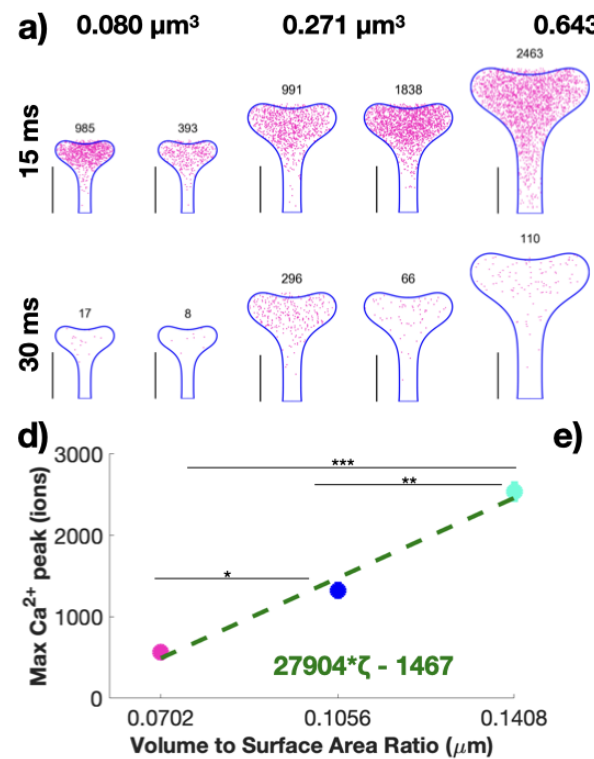

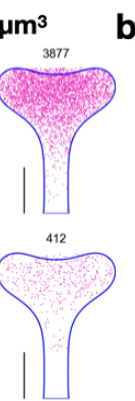

b)

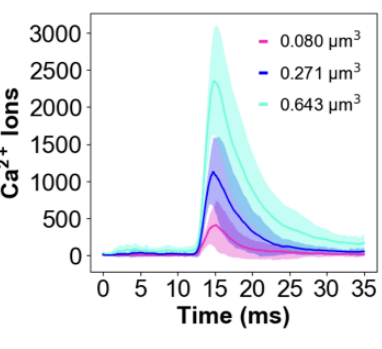

e)

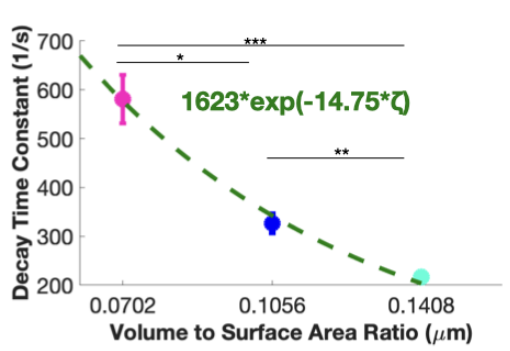

c)

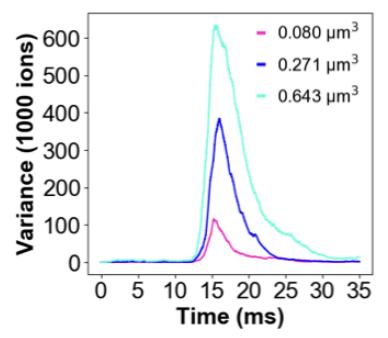

f)

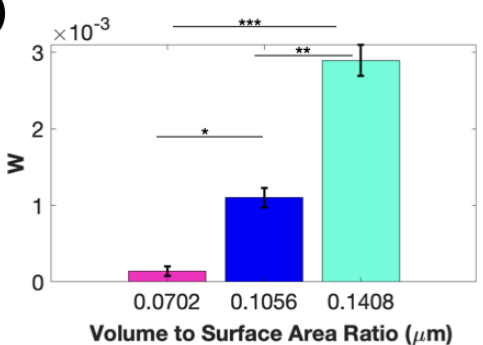

Figure 4: Changing mushroom spine size modulates calcium dynamics and synaptic weight change. a) Spatial plots illustrating $\mathrm{Ca}^{2+}$ localization at 15 and $30 \mathrm{~ms}$ for mushroom spines with different volumes $(0.080,0.271$ and $0.643 \mu \mathrm{m}^{3}$ ). The number above each geometry corresponds to the number of $\mathrm{Ca}^{2+}$ in the frame. Scale bars: $0.5 \mu \mathrm{m} . \mathrm{b}$ ) Mean (solid) and standard deviation (shaded area) of $\mathrm{Ca}^{2+}$ transients across 50 simulations for each of the three mushroom spine sizes. c) Variance of $\mathrm{Ca}^{2+}$ over time. d) The mean and standard error $(n=50)$ of the peak number of $\mathrm{Ca}^{2+}$ in different mushroom spine sizes shows statistically significant differences; $p^{*}=4.1244 \times 10^{-13} ; p^{* *}=6.6467 \times 10^{-15}$; $\mathrm{p}^{\star * *}=7.8934 \times 10^{-32}$ from two-tailed $t$-test. We fit the trend in peak $\mathrm{Ca}^{2+}$ as a linear function of volume-to-surface area ratio, $\zeta ; r^{2}=0.6655$ for the linear fit. e) The decay timescales of each $\mathrm{Ca}^{2+}$ transient are estimated by fitting with an exponential decay function $c \cdot \exp (-k t)$. The mean and standard error $(\mathrm{n}=50)$ of the decay time constant, $k$, shows statistically significant differences across mushroom spine sizes; $p^{*}=6.8175 \times 10^{-6} ; p^{* *}=6.4075 \times 10^{-6} ; p^{* \star *}=$ $1.1118 \times 10^{-10}$ from two-tailed $t$-test. The mean decay time constants as a function of volume-to-surface area ratio, $\zeta$, was fit with an exponential $a \cdot \exp (-b \zeta) ; r^{2}=0.3223$ for the exponential fit. f) The mean and standard error $(\mathrm{n}=50)$ of the calculated synaptic weight change at the last time point in the simulation for all mushroom spine sizes, plotted against the volume-to-surface area ratio, shows statistically significant differences between all cases; $p^{*}=5.1012 \times 10^{-10} ; p^{* *}$ $=2.0097 \times 10^{-11} ; \mathrm{p}^{\star \star \star}=2.1447 \times 10^{-23}$ from two-tailed $t$-test.

Finally, we repeated our analysis for mushroom-shaped spines of increasing volume $(0.080$, 
0.271 and $0.643 \mu \mathrm{m}^{3}$ ), (Figure 4). The effect of the shape of the spines is evident in the spatial dynamics of calcium (Figure $4 \mathrm{a}$ and Supplemental Movie S3). Even at $15 \mathrm{~ms}$, we note that while a vast majority of calcium ions are localized in the spine head, there is spillover of calcium into the neck; this is particularly evident in the spines of larger volume in (Figure 4a). We further investigated the role of the spine neck in both thin and mushroom spines in Figure S3 and Figure S4.

The effect of increases in volume, and therefore increases in volume-to-surface area on the temporal dynamics of calcium is an increase in peak calcium (Figure 4p,d) and variance (Figure 4c), and a decrease in the decay time constant (Figure 4e). The synaptic weight change in mushroom spines increases with spine volume-to-surface area and is larger for these mushroom spines than the filopodia-shaped and thin spines (Figure 4f). We observe that the peak calcium shows a linear increase with volume-to-surface area ratio with a slope that lies between the thin spines and filopodia-shaped spines. Finally, the decay time constant decreases with spine volume-to-surface area ratio but with a smaller exponential decay when compared to thin spines and filopodia-shaped spines. These two results point to the following conclusions - first, an increase in spine volume results in an increase in critical readouts of synaptic plasticity and second, the shape of the spine alters the quantitative relationships of synaptic plasticity by allowing access to different volume-tosurface area ratios.
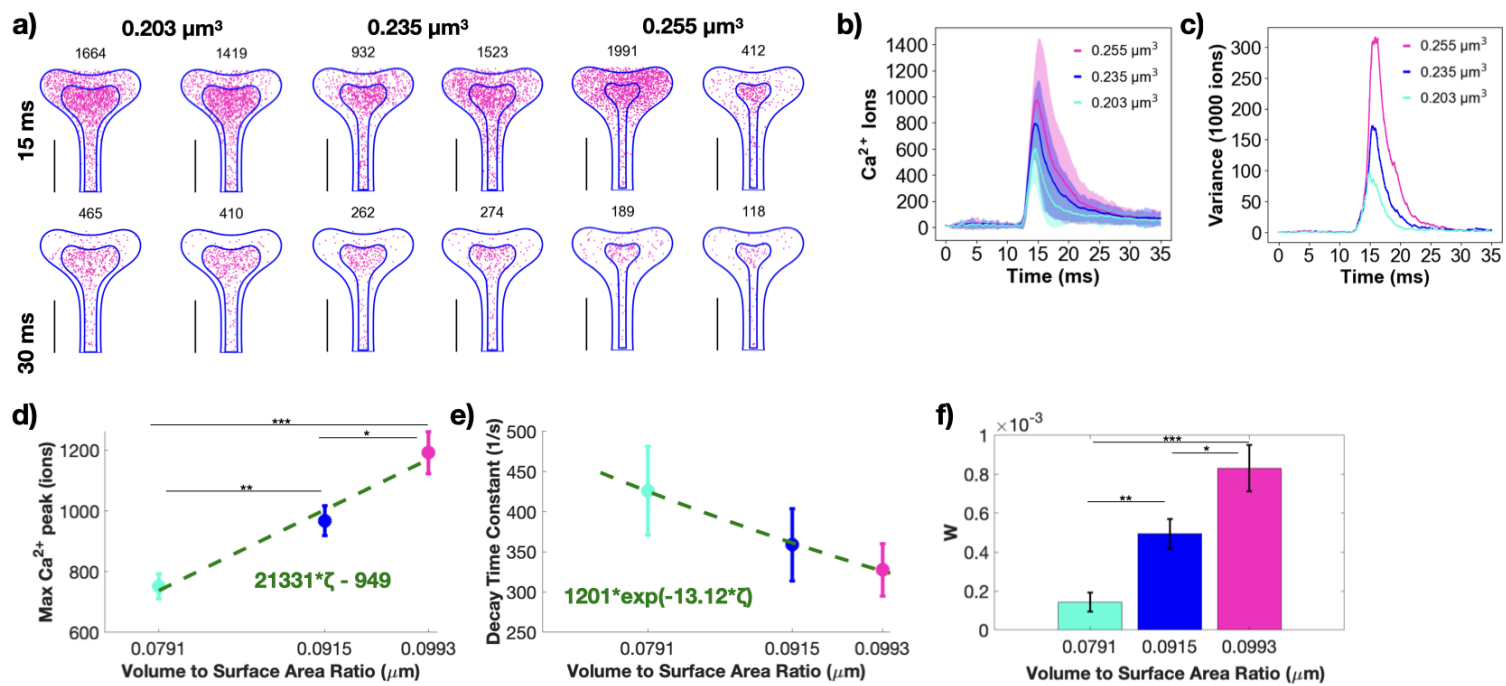

Figure 5: Spine apparatus size modulates synaptic weight change in mushroom spines. a) Spatial plots at 15 and $30 \mathrm{~ms}$ for mushroom spines with spine apparatus of different volumes (net spine volumes of $0.203,0.235$ and $0.255 \mu \mathrm{m}^{3}$ ). The numbers on top of the shape indicate the total number of calcium ions at that instant in both the spine apparatus and cytoplasm. Scale bars: $0.5 \mu \mathrm{m}$. Calcium ions over time as mean and standard deviation (b) and variance (c) for all three mushroom spines with different spine apparatus sizes. Shaded regions in (b) denote standard deviation. d) Peak calcium ion number for each mushroom spine with a spine apparatus, with the mean and standard error ( $n=50)$, show statistically significant differences; $\mathrm{p}^{*}=0.0101 ; \mathrm{p}^{* *}=0.0010 ; \mathrm{p}^{\star * *}=4.0801 \times 10^{-7}$ from two-tailed $t$-test. We fit the trend in peak values with a linear function against the volume-to-surface area ratio; $r^{2}=0.1768$ for the linear fit. e) We fit the decay dynamics of each calcium transient with $c \cdot \exp (-k t)$ and report the decay time constant, $\mathrm{k}$, as a mean and standard error $(n=50)$ against volume-to-surface area ratio. The decay time constants were not statistically different. We fit the trend in decay time constants as a function of volume-to-surface area ratio with an $\operatorname{exponential~} a \cdot \exp (-b \zeta)$, where $\zeta$ is the volume-to-surface area ratio; $r^{2}=0.0166$ for the fit. f) Calculated synaptic weight change mean and standard error $(n=50)$ at the last time point for all three mushroom spines with spine apparatus show statistically significant differences between all cases; $\mathrm{p}^{*}=0.0198 ; \mathrm{p}^{* *}=2.0977 \times 10^{-4} ; \mathrm{p}^{\star * *}=6.0097 \times 10^{-7}$ from two-tailed $t$-test. 


\subsection{Spine apparatus size tunes synaptic weight changes by altering the volume- to-surface area relationships}

Approximately $14 \%$ of dendritic spines have specialized endoplasmic reticulum called spine apparatus which are preferentially present in larger, mature spines (22, 42, 43). Furthermore, recent studies have shown that the spine apparatus and the ER are dynamic structures in the dendrite and dendritic spines (44). Previously, we showed that the spine apparatus modulates calcium transients in deterministic models of calcium influx (22) by altering the net fluxes (23). Here, we investigate how these relationships are altered in stochastic models in mushroom spines, Figure 5 (See Figure S5]for the consideration of thin spines with spine apparatus). When a spine apparatus is present in the spine head, it effectively reduces the volume of the spine cytosol and in the time frame of our consideration, acts as a calcium sink (by the action of the SERCA pumps) (45). We also varied spine apparatus size in the medium-sized mushroom spine, see Figure $5 \mathrm{a}$ and Table 4. Calcium transients and variance showed much smoother dynamics for the mushroom spines compared to the thin spines, compare Figure $5 \mathrm{~b}-\mathrm{c}$ versus Figure $55 \mathrm{~b}$-c. Peak calcium values were all statistically different for the different spine apparatus sizes and followed a linear relationship with respect to the volume-to-surface area ratio, Figure $5 \mathrm{~d}$. Decay time constants were fit with an exponential relationship but there were no statistical differences across different spines (Figure 5 e). All different spine apparatus sizes produce synaptic weight changes that are statistically different, such that increases in spine apparatus size result in smaller spine volume (and smaller volume-tosurface area ratio) and therefore produce smaller weight changes, Figure 5:. Thus, the presence of spine apparatus alters the volume-to-surface area ratio for spines and therefore tunes calcium levels and synaptic weight updates in the large mushroom spines with an inverse relationship to the spine apparatus size.

a)

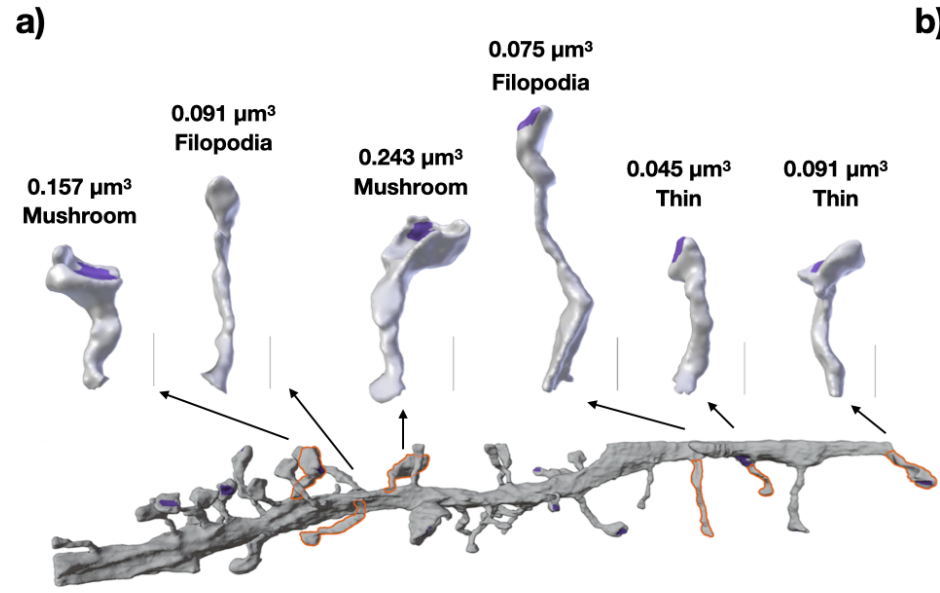

b) i)
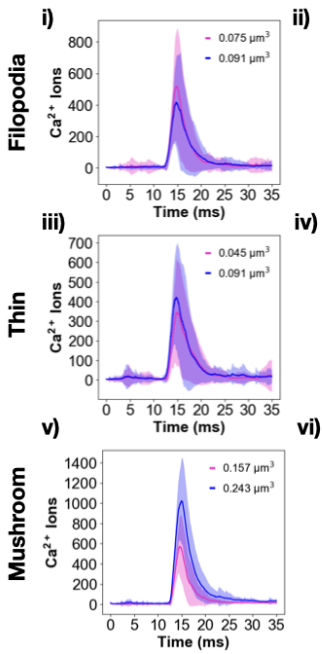
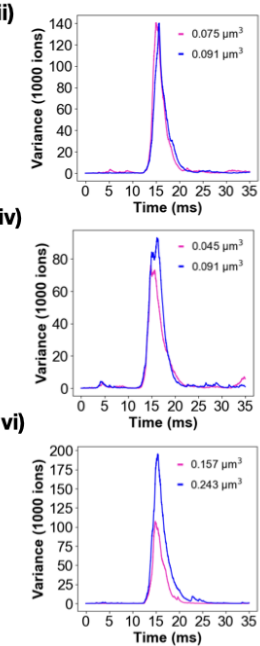

Figure 6: Real spine geometries show size dependence for calcium dynamics a) Spines similar to the idealized geometries were selected from a reconstructed dendrite (46). Representative filopodia-shaped spines, thin spines, and mushroom spines were selected and labelled with their volume and shape. Scale bars: $0.5 \mu \mathrm{m}$. b) Calcium transients as means and standard deviation, along with variance over time for the realistic spines of different shapes; i-ii) filopodiashaped spines, iii-iv) thin spines, and v-vi) mushroom spines. The realistic spines are labeled with their volumes. 


\subsection{Simulations in realistic geometries reveals that synaptic weight change de- pends on spine volume and volume-to-surface area}

Thus far, we focused on idealized geometries of spines, to identify relationships between key synaptic variables and key geometric variables. We found that the peak calcium concentration, decay time constant, and synaptic weight depend on the volume-to-surface area ratio within each shape classification. Do these relationships hold for realistic geometries as well? To answer this question, we selected realistic geometries from mesh models (47) informed by electron micrographs from Wu et al (46).

Realistic spines have more complex geometries that do not fall into the exact morphological categories that we used for idealized spines. To test the significance of these variations, we selected two spines of each shape (thin, mushroom, and filopodia-shaped) and conducted simulations with the exact same parameters as the idealized simulations (Figure 6a). We chose realistic geometries that were within the range of sizes of the idealized geometries. The PSDs in the realistic spines were annotated during the segmentation process and no modifications were made to the $P S D$ marked regions. To capture filopodia-shaped protrusions, we selected long, thin spines (with minimal differentiation between the head and neck) that had marked PSD, because we did not include dendritic filopodia in the section. Details on how to use realistic geometries in these simulation modalities can be found in the Supplemental Material.

For filopodia-shaped spines, we found that peak calcium and variance varied with volume but the variance was not appreciably different for the two spines that we used to conduct simulations (Figure 6p(i-ii), Supplemental Movie S5, Supplemental Movie S7). The realistic thin spines we chose had volumes similar to the filopodia-shaped spines and they also exhibited calcium dynamics proportional to their volume (Figure 60(iii-iv), Supplemental Movie S8, Supplemental Movie S9). Mushroom spines had larger volumes and larger PSD areas when compared to the thin or filopodiashaped spines (Figure 6o(v, vi), Supplemental Movie S4 and Supplemental Movie S6, Again, the calcium dynamics was proportional to the volume and showed that larger spines have higher peak calcium concentrations. Thus, the relationships of spine geometry and calcium dynamics hold in realistic geometries as well.

\section{Discussion}

Dendritic spines have been studied extensively as biochemical signaling compartments and their role in calcium sequestration has been theorized extensively $(2,4,22,23,48,49)$. Their unique morphological features and the classification of spine sizes and shapes with respect to function suggests possible structure-function relationships at the level of individual spines. In this work, we used stochastic modeling of calcium transients in dendritic spines of different geometries to understand how spine size and shape affect synaptic weight change. Using a stochastic simulation is important to investigate variance amongst spine shape and size as dendritic spines have small volumes and probabilistic channel dynamics. Using idealized and select realistic geometries we found that geometric properties, specifically, the volume-to-surface area affected key properties of calcium transients including peak calcium, decay time constants, and synaptic weight change. We discuss these findings in the context of different aspects of synaptic plasticity.

Our models predict despite the individual calcium transients being stochastic, there is a predictive deterministic trend that appears to carry through the different sizes and shapes of spines used in our model (Figure 7). We highlight that our goal is to demonstrate a trend in the data as opposed to building numerical functions. Although we fit the various data, we note that the $r^{2}$ is often weak, 


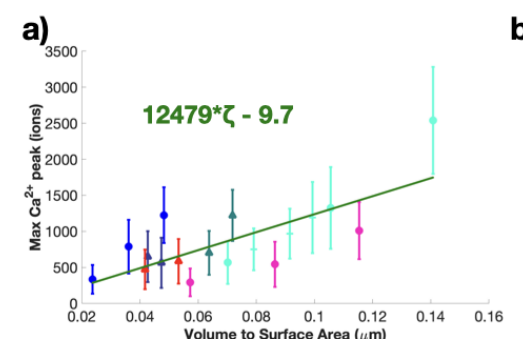

$$
\text { d) }
$$
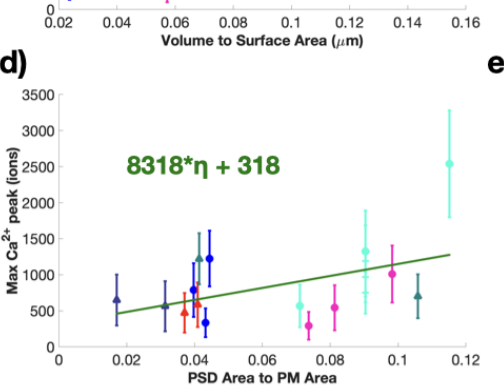

b)
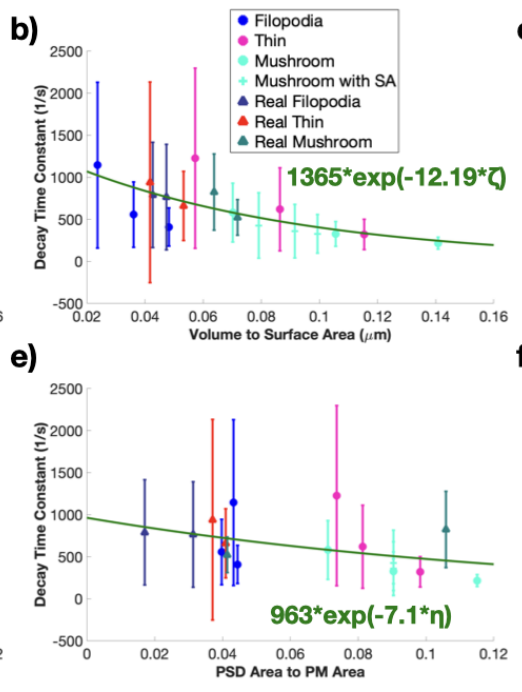

c)

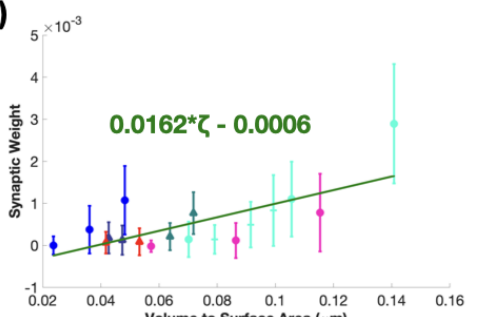

f)

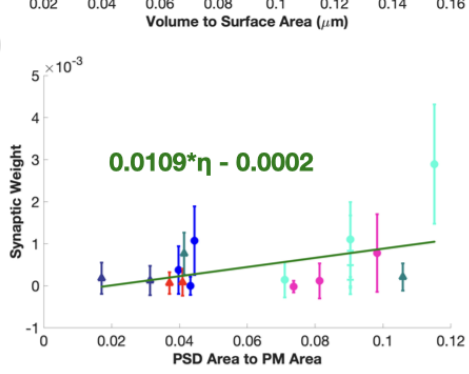

Figure 7: Idealized and realistic spines show overall trends in peak calcium, decay rates, and synaptic weight change with respect to volume-to-surface area ratios. a) All calcium peaks as mean and standard error $(n=50)$ across volume to surface area ratio show an overall increasing trend. We fit the trend in peak values with a linear function against the volume-to-surface area ratio; $r^{2}=0.351$ for the linear fit. b) We fit the decay dynamics of each calcium transient with $c \cdot \exp (-k t)$ and report the decay time constant, $\mathrm{k}$, as a mean and standard error $(\mathrm{n}=50)$ against volume-to-surface area ratio. We fit the trend in decay time constants as a function of volume-to-surface area ratio with an exponential $a \cdot \exp (-b \zeta)$, where $\zeta$ is the volume-to-surface area ratio; $r^{2}=0.1114$ for the fit. c) Calculated synaptic weight change mean and standard error $(n=50)$ at the last time point for all idealized and realistic spines shows an increasing trend. We fit the trend in synaptic weight change with a linear function against the volume-to-surface area ratio; $r^{2}=0.2815$ for the linear fit. d) All calcium peaks as mean and standard error $(\mathrm{n}=50)$ across PSD surface area to plasma membrane surface area ratio show an overall increasing trend. We fit the trend in peak values with a linear function against the PSD-to-surface area ratio; $r^{2}=0.1441$ for the linear fit. e) We fit the decay dynamics of each calcium transient with $c \cdot \exp (-k t)$ and report the decay time constant, $\mathrm{k}$, as a mean and standard error $(\mathrm{n}=50)$ against PSD-to-surface area ratio. We fit the trend in decay time constants as a function of PSD-to-surface area ratio with an exponential $a \cdot \exp (-b \eta)$, where $\eta$ is the volume-to-surface area ratio; $r^{2}=0.0428$ for the fit. f) Calculated synaptic weight change mean and standard error $(n=50)$ at the last time point for all idealized and realistic spines shows an increasing trend. We fit the trend in synaptic weight change with a linear function against the PSD-to-surface area ratio; $r^{2}=0.1186$ for the linear fit. 
indicative of the complexities that underlie such efforts. With this in mind, one of the advantages of our modeling approach here is that we can directly compare across the entire range of idealized and realistic geometries. By considering all the data from our models, for a total of 18 geometries with 50 simulations in each, we find that the peak calcium density is more-or-less linear with the volume-to-surface area ratio (Figure $7 \mathrm{a}$ ). The decay time constant for calcium transients shows an exponential decay for larger volume-to-surface ratios with quite a bit of variability for smaller ratios (Figure $7 \mathrm{~b}$ ). And finally, the synaptic weight change increases as volume-to-surface area increases (Figure $7 \mathrm{k}$ ).

In the idealized geometries, the $\mathrm{PSD}$ area is a manually-fixed proportion of the spine volume but realistic geometries do not have this artificial constraint. Therefore, we redid our analysis using PSD area-to-surface area ratios (PSD to Plasma Membrane (PM) ratio). We still found the same relationships overall (Figure $7 \mathrm{~d}$-f) but this time with clustering of data around some ratios. This indicates that the PSD area is an important additional degree of freedom for synaptic weight change that must be considered for interpretation of geometric features and using realistic geometries with boundary markings allows us to investigate this. It is important to note that there is a lot more variability in the smaller volume-to-surface area ratios suggesting the response of smaller spines may be more erratic than larger spines. This feature can work as a double-edged sword - it may provide an advantage during the development of spines or be an disadvantage in the case of loss of spines $(50,51)$.

Finally, we interpret our predictions in the context of spine shapes. Filopodia are prevalent during early synaptogenesis and can transition into dendritic spines based on synaptic activity (37). Additionally, various disease states produce modified dendritic spines that appear more like filopodia (52). The lack of significant weight changes for the smallest filopodia-shaped spine indicates that there is a volume threshold at which filopodia receive enough stimulus trigger synaptic weight change and transition towards more stable, mature dendritic spines. Importantly, the early synaptic weight changes emphasize how the increase in spine volume changes the weight outcome from LTD to LTP. This increase in synaptic weight emphasizes how an increase in spine size can push a thin spine to transition into a stable, larger mushroom spine.

The difference in peak calcium level, decay dynamics, and synaptic weight changes as different spine shapes are scanned across different sizes can also provide insight on spine shape transitions during development and maturation. Filopodia-shaped spines have larger increases in peak calcium levels and synaptic weight updates and faster decreases in decay time constants as their volume-to-surface area ratios and volumes increase, compared to both thin and mushroom spines; Figure 2, Figure 3 , and Figure 4. This suggests that filopodia can very quickly alter their calcium levels, and therefore are well-suited for initially identifying possible synaptic partners and subsequently directing resources to those filopodia that are good candidates to transition to dendritic spines (53). Once filopodia are established, their linear calcium increase with volume might be unsustainable and might lead to the reduced levels of increase for thin spines of comparable volume-to-surface area (and volume). This suggests that larger stimuli might be necessary to push thin spines towards more excitation, perhaps prevent excessive numbers of thin spines from maturing and leading to resource depletion and excess neural connectivity (54). Mushroom spines once again show more of an increase in synaptic weight as they increase in volume-to-surface area ratio (and volume) but at volumes shifted from the filopodia-shaped spines, perhaps highlighting their role as key communication hubs (54). The volume shift seen in mushroom spines versus filopodiashaped spines might serve to limit the number of mature, highly excitable dendritic spines as both a key neuronal network and resource regulation feature. When the spine apparatus acts as a sink, its presence dampens synaptic weight changes in mushroom spines, potentially acting to stabilize the spine from future changes as suggested by others $(41,55)$. 
We note that our study is only a small piece of the puzzle with respect to synaptic plasticity. For instance, whether one should use total number of calcium ions or use calcium concentration in evaluating synaptic weight change requires additional exploration. For instance, we find that when calcium results are converted from total ions to average concentration along with the phenomenological synaptic weight equations, we get different trends in synaptic weight update results, Figure 57. However, converting our previous results (22) into total ions shows the same trends for max $\mathrm{Ca}^{2+}$ peak and decay time constants as this current study, Figure S6. Thus, a simple unit issue can lead to conflicting results in spatial models and indicates that we need further discussion and investigation on the structure of phenomenological equations for synaptic weight to understand which factors of calcium dynamics matter and to what degree. An additional limitation of this study is the usage of traditional p-values for statistical analysis of the data (see Figure $S 8$ for details on $h$ and $p$ values), since the statistics field has suggested moving away from null-hypothesis significance testing (56). We also note that our current focus is on very early events and these models must be extended to longer time scale events to explore the biochemical and geometric interplay for downstream signaling $(57-60)$.

In summary, our computational models using idealized and realistic geometries of dendritic spines have identified potential relationships between spine geometry and synaptic weight change that emerge despite the inherent stochasticity of calcium transients. The advances in computational modeling and techniques have set the stage for a detailed exploration of biophysical processes in dendritic spines (57,61, 62). Such efforts are critical for identifying emergent properties of systems behavior and also eliminating hypotheses that are physically infeasible (63, 64). Models such as this and others can set the stage for investigating longer time scale events in spines including the downstream effectors of calcium (16, 58, 65, 66), and actin remodeling for structural plasticity $(67,68)$.

\section{Methods}

We developed a stochastic reaction diffusion model in MCell (33). The reactions are obtained from Bartol et al. (24) and Bell et al. (22) and are discussed in detail below.

\subsection{Simulation Information and Parameters}

Simulations were run for a total simulation time of $35 \mathrm{~ms}$ with a $500 \mathrm{~ns}$ time step. Each geometry is simulated in MCell over 50 distinct seeds to generate an appropriate sample size of results, and we use a write-out frequency of once per iteration to allow for reproducibility of results. At the beginning of each simulation, membrane proteins are distributed randomly over specified regions of the spine geometry surface area according to an assigned count or concentration. The reaction rates for all components in the model system were adjusted in (24) to reflect a system temperature between $34^{\circ} \mathrm{C}$ and $37^{\circ} \mathrm{C}$.

\subsection{A Note About the Treatment of Extracellular Calcium}

Extracellular calcium was not explicitly modeled for ease of computational tractability. We assumed a constant extracellular calcium concentration that is negligibly impacted by the calcium influx to and efflux from the spine cytoplasm. The dynamics of $\mathrm{Ca}^{2+}$ ions are explicitly modeled once they enter the cell through channels located on the $\mathrm{PM}$, and cease to be explicitly represented once they are pumped out of the cell. 


\subsection{Dynamics of calcium ions in the spine volume}

We summarize the main reactions for $\mathrm{Ca}^{2+}$ ions in the volume. The values for the reaction rates and other important model parameters are located in Table 1. In the spine volume, calcium decay took the form given below where $k_{d}$ sets a decay time scale,

$$
\mathrm{Ca}^{2+} \stackrel{\mathrm{k}_{d}}{\longrightarrow} \varnothing \text {. }
$$

The value of $\mathrm{k}_{d}$ is taken as $50 \mathrm{~s}^{-1}$ based on (22). We note that this is significantly smaller than the decay rate constant determined in the results. This is expected as this cytosolic calcium decay is just one means of calcium clearing from the cytoplasm, along with the various pumps, and mobile and fixed buffers.

In the volume, calcium binds with fixed and mobile buffers in the cytoplasm, modeled here generically with $\mathrm{B}_{m}$ to represent mobile calcium buffers, and $\mathrm{B}_{f}$ to represent fixed buffers. Calciumbuffer binding is modeled in MCell with the reactions

$$
\mathrm{Ca}^{2+}+\mathrm{B}_{f} \underset{\mathrm{k}_{\mathrm{Bf}, \text { off }}}{\stackrel{\mathrm{kff} \text { on }}{\rightleftarrows}} \mathrm{Ca} \cdot \mathrm{B}_{f}
$$

and

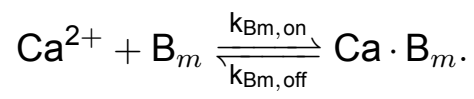

Reaction rates for the mobile and fixed buffers are found in Table 1.

Table 1: Parameters used in the model for volume.

\begin{tabular}{cccc}
\hline Variable & Value & Units & Reference \\
\hline Init. $\left[\mathrm{Ca}^{2+}\right]_{\mathrm{cyto}}$ & $1 \times 10^{-7}$ & $\mathrm{M}$ & $(24,69)$ \\
Init. $\left[\mathrm{Ca}^{2+}\right]_{\mathrm{ER}}$ & $6 \times 10^{-5}$ & $\mathrm{M}$ & $(24)$ \\
Init. $\left[\mathrm{Ca}^{2+}\right]_{\mathrm{ECS}}$ & 2 & $\mathrm{mM}$ & $(\overline{\overline{70}}$ \\
$k_{d}$ & 50 & $\mathrm{~s}^{-1}$ & $(22)$ \\
Init. $\left[\mathrm{B}_{f}\right]$ & $2 \times 10^{-5}$ & $\mathrm{M}$ & $(\overline{71})$ \\
Init. $\left[\mathrm{B}_{m}\right]$ & 4791 & molecule $\mu \mathrm{m}^{-2}$ & $(24)$ \\
\hline
\end{tabular}

\subsection{Plasma Membrane}

The primary influx of calcium through the plasma membrane occurs through NMDARs andVSCCs and calcium is pumped out of the cell via two kinds of pumps: PMCA and NCX In this model, NMDARs are both voltage and glutamate dependent and are localized to the PSD region. VSCCS are voltage dependent and located throughout the plasma membrane surface. $\mathrm{PMCA}$ and $\mathrm{NCX}$ are calcium-dependent pumps and are also located throughout the plasma membrane surface.

\subsubsection{NMDA receptors}

NMDAR are localized to the PSD area with areal density 150 molecule $\mu \mathrm{m}^{-2}(24)$. The activation of NMDAR is modeled with an asymmetric trapping block kinetic scheme as proposed by Ref. (72). The activation of NMDAR is dependent on the diffusion of glutamate through the synaptic cleft, 
and its binding to inactive receptors. In this study, a surface identical to the top of the spine head is displaced $2 \mu \mathrm{m}$ above the head, approximating the synaptic cleft. At time $t=0$ in each simulation, 500 molecules of glutamate are released at the center of this synaptic cleft at the beginning of simulation, and subsequently diffuse through the space at a rate of $2.2 \times 10^{-6} \mathrm{~cm}^{2} \mathrm{~s}^{-1}$, where they bind to membrane-bound proteins. On the postsynaptic membrane, NMDARs compete with the glutamate receptor AMPAR for glutamate; thus, AMPARs are also included in the simulation to model this competition but they do not play a role in calcium influx. AMPAR is also localized to the PSD area. The binding of glutamate to AMPAR is modeled according to the kinetic scheme proposed by Ref. (73).

Calcium flux through open NMDARs is modeled in MCell with a simple monomolecular reaction.

$$
\text { NMDAR } \stackrel{\mathrm{k}_{\mathrm{Ca}}}{\longrightarrow} \mathrm{NMDAR}+\mathrm{Ca}_{\text {cyto }}^{2+}
$$

where the rate of calcium influx is given by

$$
\mathrm{k}_{\mathrm{Ca}}(V)=\gamma_{N M D A R} \cdot \frac{V-V_{r}}{2 \cdot 1.6 \times 10^{-19}} .
$$

$\mathrm{V}$ is the membrane potential, and $\mathrm{V}_{\mathrm{r}}$ is the reversal potential for NMDAR. The parameters for the NMDAR reactions are the same as given in (72) and the parameters for the AMPAR reactions are the same as given in Ref. (73).

\subsubsection{Calcium influx through voltage-sensitive calcium channels}

The influx of $\mathrm{Ca}^{2+}$ through an open VSCC is given by the reaction:

$$
\text { VSCC } \stackrel{\text { kvscc }}{\longrightarrow} \text { VSCC }+\mathrm{Ca}_{\text {cyto }}^{2+}
$$

where the rate of calcium influx is given by

$$
\mathrm{k}_{\mathrm{Vscc}}=\frac{\gamma V(t) N_{A}\left[0.393-\exp \left(\frac{-V(t)}{80.36}\right)\right]}{2 F\left[1-\exp \left(\frac{V(t)}{80.36}\right)\right]} .
$$

The influx of $\mathrm{Ca}^{2+}$ through VSCCs is also dependent on the activation kinetics of VSCCs. The initial conditions for all the VSCCS is the closed state, and the activation of the channels is modeled here with a five state kinetic scheme as used in Ref. (24). The parameters for $\mathrm{Ca}^{2+}$ influx through VSCCs are the same as in (24). We included a VSCCs density of 2 molecule $\mu \mathrm{m}^{-2}$.

\subsubsection{Voltage calculations in the model}

Since the transmembrane potential is time-varying and the rate constants for NMDAR and VSCC are voltage-dependent, the values of these rate constants at each simulation step were pre-computed and passed into MCell. The voltage stimulus representing a single EPSP starting at time $t=0$, followed by a single BPAP occurring at an offset of $10 \mathrm{~ms}$ was obtained from Ref. (24). Note that this time offset is within the typical window for Spike-Timing Dependent Plasticity (STDP) to inducing LTP (24, 74).

\subsubsection{PMCA and NCX}

PMCA and NCX are located on the plasma membrane with areal density 998 molecule $\mu \mathrm{m}^{-2}$ and 142 molecule $\mu \mathrm{m}^{-2}$ respectively (24), forcing an efflux of calcium out of the cell. These pumps are modeled using the set of elementary reactions and reaction rates from Ref. (24). 


\subsubsection{Spine Apparatus}

Calcium enters the spine apparatus via SERCA pumps, and exits by leakage. SERCA pumps are

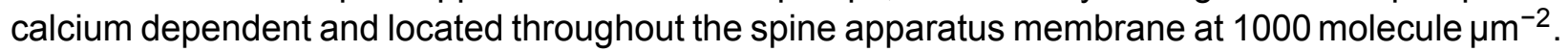
SERCA influx is modeled as a series of elementary reactions with rates from Ref. (24). Calcium leakage from the spine apparatus into the cytosol is modeled by the reaction

$$
\mathrm{Ca}_{\mathrm{ER}}^{2+} \stackrel{k_{\text {leak }}}{\longrightarrow} \mathrm{Ca}_{\text {cyto }}^{2+},
$$

where $k_{\text {leak }}$ is $0.1608 \mathrm{~s}^{-1}$ from Ref. (22).

\subsection{Synaptic weight change}

We considered the effects of a single instance of spine activation on cytosolic calcium dynamics and subsequent synaptic weight change. Therefore, we can interpret this synaptic weight change as an early indicator of longer synaptic weight changes. We modeled changes in synaptic weight, $w$, due to cytosolic calcium as a phenomenological relationship, inspired by (7, 55). Synaptic weight change is given by

$$
\frac{d w}{d t}=\frac{\Omega_{w}-w}{\tau_{w}},
$$

where $\tau_{w}$ is a learning rate given as

$$
\tau_{w}=k_{1}+\frac{k_{2}}{k_{3}+2 \mathrm{Ca}_{\text {cyto }}^{2+}(t) /\left(\theta_{D}+\theta_{P}\right)},
$$

and $\Omega_{w}$ describes calcium dependence in the regimes of [LTP and [LTD as

$$
\Omega_{w}=\frac{1}{1+\exp \left(-\beta_{P}\left(\mathrm{Ca}_{\mathrm{cyto}}^{2+}(t)-\theta_{P}\right)\right)}-\frac{0.5}{1+\exp \left(-\beta_{D}\left(\mathrm{Ca}_{\mathrm{cyto}}^{2+}(t)-\theta_{D}\right)\right)} .
$$

Cytosolic calcium, $\mathrm{Ca}_{\text {cyto }}^{2+}(t)$, is input as total ions in the spine in the above equation. The differential equation for synaptic weight, $w$, is solved in MATLAB 2018b using ode23s, with an initial synaptic weight value of 0 so synaptic weight change and synaptic weight are the same value for this single stimulation event. Synaptic weight parameters are given in Table 2.

Because we are working with a stochastic model and are considering $\mathrm{Ca}^{2+}$ in terms of ions, we converted the parameters in the synaptic weight equations from units involving concentration to units of molecules, based on average spine volumes and realistic numbers of calcium ions in dendritic spines. It is important to note that using total $\mathrm{Ca}^{2+}$ ions is a global view of the dendritic spine while concentration can be considered as more of a local measurement. As mentioned, this synaptic weight change is a phenomenological relationship between $\mathrm{Ca}^{2+}$ and synaptic weight which captures the concept of synaptic strength change, and it remains unclear if using ions versus concentration is a better approach for predicting this change. We converted our results into average concentrations by dividing the calcium transients by the respective spine volume, converting our synaptic weight parameters into units of concentration, and rerunning our synaptic weight calculations, Figure S7. Further investigation is required to understand the considerations behind these different approaches. 
Table 2: Parameters for Synaptic Weight.

\begin{tabular}{|c|c|c|c|}
\hline Variable & Value & Units & Reference \\
\hline Init. w & 0 & - & (55) \\
\hline$k_{1}$ & 1 & $\mathrm{~s}$ & (55) \\
\hline$k_{2}$ & 10 & $\mathrm{~s}$ & $(\overline{55})$ \\
\hline$k_{3}$ & $1 \times 10^{-3}$ & - & (55) \\
\hline$\theta_{D}$ & 100 & molecule & $*(\sqrt{7}, 55)$ \\
\hline$\theta_{P}$ & 400 & molecule & $*(7, \overline{75})$ \\
\hline$\beta_{D}$ & 0.2977 & molecule ${ }^{-1}$ & $*(7,55)$ \\
\hline$\beta_{P}$ & 0.2977 & molecule $^{-1}$ & $*(\overline{\overline{7}}, \overline{\overline{55}})$ \\
\hline
\end{tabular}

* These parameters were converted from concentration units with adjustments for consistency.

\subsection{MATLAB Analysis of $\mathrm{Ca}^{2+}$ transients}

We used MATLAB version $2018 \mathrm{~b}$ to analyze the max $\mathrm{Ca}^{2+}$ peak and decay time constants for the stochastic $\mathrm{Ca}^{2+}$ results. For each realization of the $\mathrm{Ca}^{2+}$ transient, we used the $\max ()$ function to find the peak $\mathrm{Ca}^{2+}$ value and corresponding time. We fit the transient after the peak using the fit () function set to 'exp1'. The parameters from each fit, corresponding to a realization from a random seed, and statistics such as the mean and standard deviations are computed. The standard error of the mean was found by dividing the standard deviation by the square root of the number of individual trials, in this case 50 trials.

\subsection{Statistical Analysis}

Statistical significance was determined using a two-tailed two-sample $t$-test assuming equal means and variance (ttest2() function) in MATLAB version 2018b with a significance cutoff at $p=0.05$. Statistical comparisons were made between the distributions of observables yielded by the 50 simulations of the compared experimental conditions. Trends in the stochastic results data were fit using all 50 seeds for each of the simulations being considered in the fit. The reported trend lines are estimated using the data from all 50 seeds, as opposed to fitting to the means only. Linear fits and exponential fits were computed in MATLAB using the functions fitlm() and fit(), respectively. We highlight that we are using the classical approach of null-hypothesis significance testing, p-values, and statistically significant verbiage, which has been questioned as perilous and oversimplistic (56). We have provided the $p$-values for each result comparison for closer consideration, Figure S8. The linear and exponential trend lines shown have a range of $r^{2}$ values and are used to show general trends. We emphasize however that in some plots we are fitting to either very few data points or a small domain. Therefore, we reiterate that these factors limit the interpretation of the quantitative nature of the fits. 


\section{Geometries}

Idealized, axisymmetric geometries are used to represent the structure of dendritic spines in this study. Three general spine shapes are represented - thin, mushroom, and filopodia-shaped - and each shape is further varied in size and, for the thin and mushroom spines, neck radius.

\subsection{Geometry generation}

The geometries were generated from 2-dimensional ideal spine profiles obtained from Ref. (36) consisting of a series of points $(r, z)$ which form the outline of the respective geometry's rotational cross-section. Using Netgen/NGSolve version $6.2(75)$, we revolved these profiles about the z-axis to yield a rotationally-symmetric 3-dimensional spine geometry, Figure 8. In all spine geometries, a circular PSD was centered at the top of the spine head. The PSD area was set as a function of spine volume according to the relationship observed in Ref. (76).

\subsection{Size and neck variations}

To further explore the effects of geometric variations on calcium transients and stochasticity, and to facilitate the comparison of spine geometries of similar volumes and different shapes, the base geometries of all three shapes are scaled to two additional volumes beyond the base shapes from (36). The additional versions of the thin spine, initially smaller than the other spine shapes, are scaled such that their length measurements are 1.5 and 2 times their original values, resulting in volumes 3.375 and 8 times that of the initial thin spine, respectively. The base mushroom spine, intermediate in volume, is scaled to 0.66 and 1.33 times its original size, resulting in volumes 0.287 and 2.353 times their original value, respectively. And the base filopodia-shaped spine, initially the largest in volume, is scaled to 0.5 and 0.75 times its original size, resulting in volumes 0.125 and 0.422 times the original volume. This scheme ultimately results in three different sizes for each spine shape, spanning a similar range of volumes.

The neck radius of the thin and mushroom spines is also varied, with neck length modified as well to preserve spine volume. To create the different spine sizes, the 2-dimensional spine profiles are dilated about the origin by a certain scale factor, and the resultant image is rotated about its vertical axis using Netgen/NGSolve to produce a scaled-up or scaled-down three-dimensional geometry. In the thin and mushroom 2-dimensional profiles, the $\mathrm{x}$-values of points along the spine neck are scaled by a certain coefficient, and the length of the neck is then scaled by the squared inverse of the coefficient in order to maintain an approximately constant volume. A list of all spine geometries used, and their respective geometric measures, is found in Table 3.

\subsection{Spine Apparatus}

Some dendritic spines are observed to have a spine apparatus denoted as SpApp an extension of the smooth endoplasmic reticulum, extending from the dendrite into the neck and head of the spine (43). In this study, the effects of the presence of the SpApp on calcium transients and stochasticity are investigated; to achieve this, the thin and mushroom spine geometries are further modified with the addition of a spine apparatus of varying sizes. For both spine shapes, the control-sized SpApp geometry is constructed by scaling down the original spine geometry and extending the spine apparatus neck, such that the SpApp occupies approximately $10 \%$ of the spine volume and extends to the base of the spine. SpApp size is then varied by scaling the SpApp geometry up and down, changing the neck length such that the SpApp base coincides with the spine base. SpApp 
2-Dimensional Profile

a)

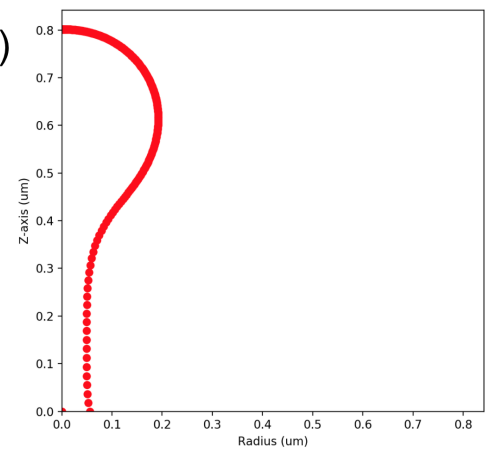

b)

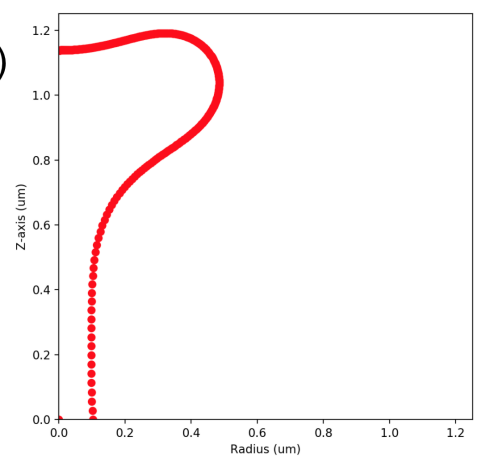

c)

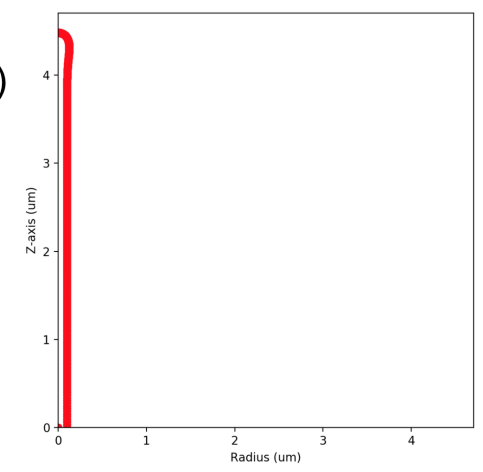

Spine Geometry
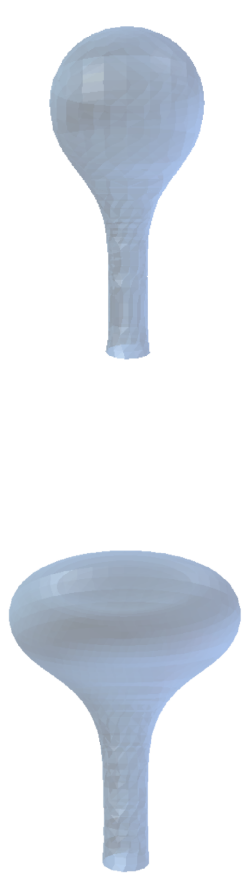

Figure 8: The 2-dimensional spine profiles and the resultant rotationally-symmetric spine geometries for a) thin spines, b) mushroom spines, and c) filopodia. 


\begin{tabular}{|c|c|c|c|c|c|}
\hline Geometry & Scale & Volume $\left(\mu \mathrm{m}^{3}\right)$ & Surface Area $\left(\mu \mathrm{m}^{2}\right)$ & Neck Radius ( $\mu \mathrm{m})$ & PSD Area $\left(\mu \mathrm{m}^{2}\right)$ \\
\hline \multicolumn{6}{|l|}{ Thin } \\
\hline small & $x 1$ & 0.035 & 0.611 & 0.06 & 0.045 \\
\hline thin neck & $x 1$ & 0.034 & 0.653 & 0.04 & 0.045 \\
\hline thick neck & $x 1$ & 0.035 & 0.590 & 0.07 & 0.045 \\
\hline medium & $\times 1.5$ & 0.119 & 1.378 & 0.08 & 0.112 \\
\hline large & $x 2$ & 0.283 & 2.453 & 0.11 & 0.241 \\
\hline \multicolumn{6}{|l|}{ Mushroom } \\
\hline small & $x 0.67$ & 0.080 & 1.140 & 0.07 & 0.081 \\
\hline medium & $x 1$ & 0.271 & 2.567 & 0.10 & 0.232 \\
\hline thin neck & $x 1$ & 0.270 & 2.689 & 0.08 & 0.232 \\
\hline thick neck & $x 1$ & 0.272 & 2.507 & 0.13 & 0.232 \\
\hline large & $x 1.33$ & 0.643 & 4.568 & 0.13 & 0.526 \\
\hline \multicolumn{6}{|c|}{ filopodia-shaped } \\
\hline small & $x 0.5$ & 0.017 & 0.717 & 0.05 & 0.031 \\
\hline medium & $x 0.75$ & 0.058 & 1.609 & 0.08 & 0.064 \\
\hline large & $x 1$ & 0.138 & 2.860 & 0.10 & 0.127 \\
\hline
\end{tabular}

is not added to the filopodia-shaped geometry, as the spine apparatus is not generally found to be present in such spine shapes (43). The SpApp-containing geometries are also listed in Table 4.

Table 3: A list of all geometric variations.

Table 4: A list of spine apparatus variations.

\begin{tabular}{llcc}
\hline Geometry & SA size & SA Volume $\left(\boldsymbol{\mu m}^{\mathbf{3}}\right)$ & Cytoplasm Volume $\left(\boldsymbol{\mu m}^{\mathbf{3}}\right)$ \\
\hline \multirow{3}{*}{ Thin } & Small & 0.00211 & 0.033 \\
& Medium & 0.00465 & 0.030 \\
& Large & 0.00867 & 0.026 \\
\multirow{2}{*}{ Mushroom } & Medium & 0.0358 & 0.255 \\
& Small & 0.0160 & 0.235 \\
& Large & 0.0676 & 0.203 \\
\hline
\end{tabular}

\subsection{Realistic Geometries}

Realistic geometries were chosen from among those on the full dendrite geometry generated in Ref. (47). Briefly, the geometric meshes were generated from electron micrographs in Wu et al. (46) using GAMer 2 (77). Individual spines with labeled PSD and volumes similar to the idealized geometries were selected from the realistic dendritic branch. 
Table 5: Table of values for realistic geometries.

\begin{tabular}{ccccc}
\hline Spine Number & Shape & Volume $\left(\boldsymbol{\mu m}^{\mathbf{3}}\right)$ & Surface Area $\left(\boldsymbol{\mu m}^{2}\right)$ & PSD Area $\left(\boldsymbol{\mu m}^{\mathbf{2}}\right)$ \\
\hline 13 & Mushroom & 0.157 & 2.457 & 0.26 \\
17 & Filopodia & 0.091 & 1.916 & 0.06 \\
18 & Mushroom & 0.243 & 3.383 & 0.14 \\
37 & Filopodia & 0.075 & 1.756 & 0.03 \\
39 & Thin & 0.045 & 1.078 & 0.04 \\
41 & Thin & 0.091 & 1.710 & 0.07 \\
\hline
\end{tabular}

\section{Acknowledgements}

This work was supported by a National Defense Science and Engineering Graduate (NDSEG) Fellowship to M.K.B., a Hartwell Foundation Postdoctoral Fellowship to C.T.L., and Air Force Office of Scientific Research FA9550-18-1-0051 to P.R.. MCell development is supported by the NIGMSfunded (P41-GM103712) National Center for Multiscale Modeling of Biological Systems (MMBioS). 


\title{
Supplemental Material for Stochastic simulations reveal that dendritic spine morphology regulates synaptic plasticity in a deterministic manner
}

\author{
M. V. Holst*, M. K. Bell*, C. T. Lee, and P. Rangamani** \\ Department of Mechanical and Aerospace Engineering, \\ University of California San Diego, La Jolla CA 92093. \\ *Both these authors contributed equally \\ **To whom correspondence must be addressed: prangamani@ucsd.edu
}

\section{S1 Additional simulation results}

\section{S1.1 Simulation results versus other geometric parameters show various trends}

We plot max $\mathrm{Ca}^{2+}$ peak, decay time constant, and synaptic weight against volume for all size variations of filopodia-shaped spines, thin spines, mushroom spines, and mushroom spines with spine apparatus Figure S1. We see similar trends across volume as we observe across volumeto-surface area ratio. We plot all results together on the same plot for max $\mathrm{Ca}^{2+}$ peak, decay time constant, and synaptic weight against volume-to-PSD area and volume, Figure S2. We see almost no dependence on volume-to-PSD area for any of the readouts. We see similar trend versus volume as we see in volume-to-surface area ratio.

\section{S1.2 Spine neck size shows differences in the large mushroom spines but not the smaller thin spines}

The spine neck has long been discussed as a key parameter governing calcium signaling within dendritic spines (25). We also explored the effects of varying spine length and radius, while preserving spine volume. We first varied the spine neck on thin spines of the control volume, Figure S3a. We saw that while the calcium transients have considerable overlap, the thin-necked spine shows significant variance at later time points compared to the other spines, Figure S3b-c. We see no statistically significant differences between peak calcium values and only decay differences between the thinnest and thickest necks, Figure S3d-e. Synaptic weight changes for the thin spines with different neck geometries showed no significant differences but were trended towards negative weight changes for thicker necks, Figure S3: We next explored mushroom spines with thinner or thicker neck geometries but with the same volume as the mushroom control spine, Figure S4a. While the mean of the calcium transients appeared quite close, there was significant difference in variance for the mushroom spine with the thick neck, Figure S4p-c. We saw differences in peak calcium only between the thinnest and thickest of the mushroom neck cases, and no significant difference in decay time constant, Figure S4d-e. Synaptic weight calculations show that presence of the thinnest versus thickest neck on a mushroom spine does lead to statistically significant differences in synaptic weight updates, Fig. S4F. This indicates that spine neck morphology might have more implications for these larger mushroom spines, compared to the smaller thin spines. 


\section{S1.3 The presence of spine apparatus in thin spines cause no clear trend in synap- tic weight update}

We vary the size of spine apparatus in thin control spines with the spine apparatus acting as a calcium sink with SERCA pumps, Figure $\mathrm{S5}$ a. We see that the presence of spine apparatus makes the calcium transient response more complex with a double peak visible in the variance for thin spines, Figure $55 \mathrm{p}-\mathrm{c}$. While we can fit the peak calcium values and decay time constant trends against both volume (Figure $S 5 \mathrm{~d}, \mathrm{e}$ ) and volume-to-surface area ratio (Figure $\mathrm{S5g}, \mathrm{h}$ ), spine apparatus presence shows no clear trend in synaptic weight change for thin spines and the differences were not statistically significant, Figure S5F.

\section{S1.4 Our previous deterministic results match the qualitative trends seen in these results}

We previously published a deterministic reaction diffusion model of calcium dynamics in dendritic spines of different morphologies (22). We found trends in the peak calcium concentration over spine volumes in that work and wanted to directly compare those results to our findings in this work. Using the results from (22), we integrate calcium concentration over the spine volume at each time point and find the peak calcium in ions and fit the decay dynamics of the calcium transient with an exponential decay function, $c \cdot \exp (-k t)$. We compare the peaks and decay time constants over both volume and volume-to-surface area ratio, and find the same qualitative trends as our findings in this currents work, Figure S6.

\section{S1.5 Synaptic weight changes depends on calculations with ions versus concen- tration}

Synaptic weight update equations are typically phenomenological relationships based on $\mathrm{Ca}^{2+}$. Historically, many mathematical models considering synaptic weight changes have considered synaptic weight changes in terms of concentration $(6,7,55)$. In this model, we consider $\mathrm{Ca}^{2+}$ in terms of $\mathrm{Ca}^{2+}$ ions. We want to consider if the use of ions versus concentration influences the synaptic weight update results. We converted the synaptic weight equations by converting the parameters from units involving molecules to concentration by dividing by the average spine volume $\left(0.09 \mu \mathrm{m}^{3}\right)$ and converting to $\mu \mathrm{M}$. We convert all the $\mathrm{Ca}^{2+}$ transients to $\mu \mathrm{M}$ by dividing by each respective spine geometry volume and modifying units. We plot the synaptic weight change at $35 \mathrm{~ms}$ for all simulations when considering ions versus concentration Figure S7. We see that synaptic weight change does change between using ions versus concentration because the concentration also considers the volume of the spines. Using concentration leads to a decreasing trend in synaptic weight with increasing volume which is the opposite of the trend seen using ions. We do however still see protrusion-type specific trends within the overall dynamics. There are several considerations to make during this comparison. First, as mentioned, the synaptic weight equations used are phenomenological relationships between $\mathrm{Ca}^{2+}$ and the concept of synaptic weight which captures the idea of synaptic strengthening which would actually occur through the insertion of receptors, such as AMPAR, and potentially spine volume increase. It remains unclear if total ion count, which is a global consideration of the whole spine, or $\mathrm{Ca}^{2+}$ concentration, which considers the local environment, is the correct value to consider for synaptic weight calculations. Furthermore, we used average concentration in Figure S7k-d) but dendritic spines are known to have signaling nanodomains, so it could be possible that it would be more accurate to consider peak concentration instead of average concentration for this calculation. Additionally, it is possi- 
ble that the thresholds for LTP versus LTD need to be modified for considering a global reading, such as total ions in the spine, versus a local measurement, such as local concentration. Should synaptic weight change depend on the total amount of $\mathrm{Ca}^{2+}$ influx or the local environment within the spine? This is an ongoing consideration that needs further analysis and discussion.

\section{S1.6 Two-tailed $t$-test results for all stochastic simulations}

We conduct two-tailed $t$-test calculations between all stochastic simulations for both idealized and real geometries for max $\mathrm{Ca}^{2+}$ peak, decay time constant, and synaptic weight change. We display both the $h$ and $p$ value for each comparison, Figure 58 . We use a $p$ threshold of 0.05 to determine the binary $h$ value. A p value smaller than 0.05 indicates that the two results are statistically different and produce a h-value of 1 . Reversely, a $p$ value larger than 0.05 indicates that the two results are not statistically different and produce a $h$-value of 0 . p-values have been truncated at two decimal points.

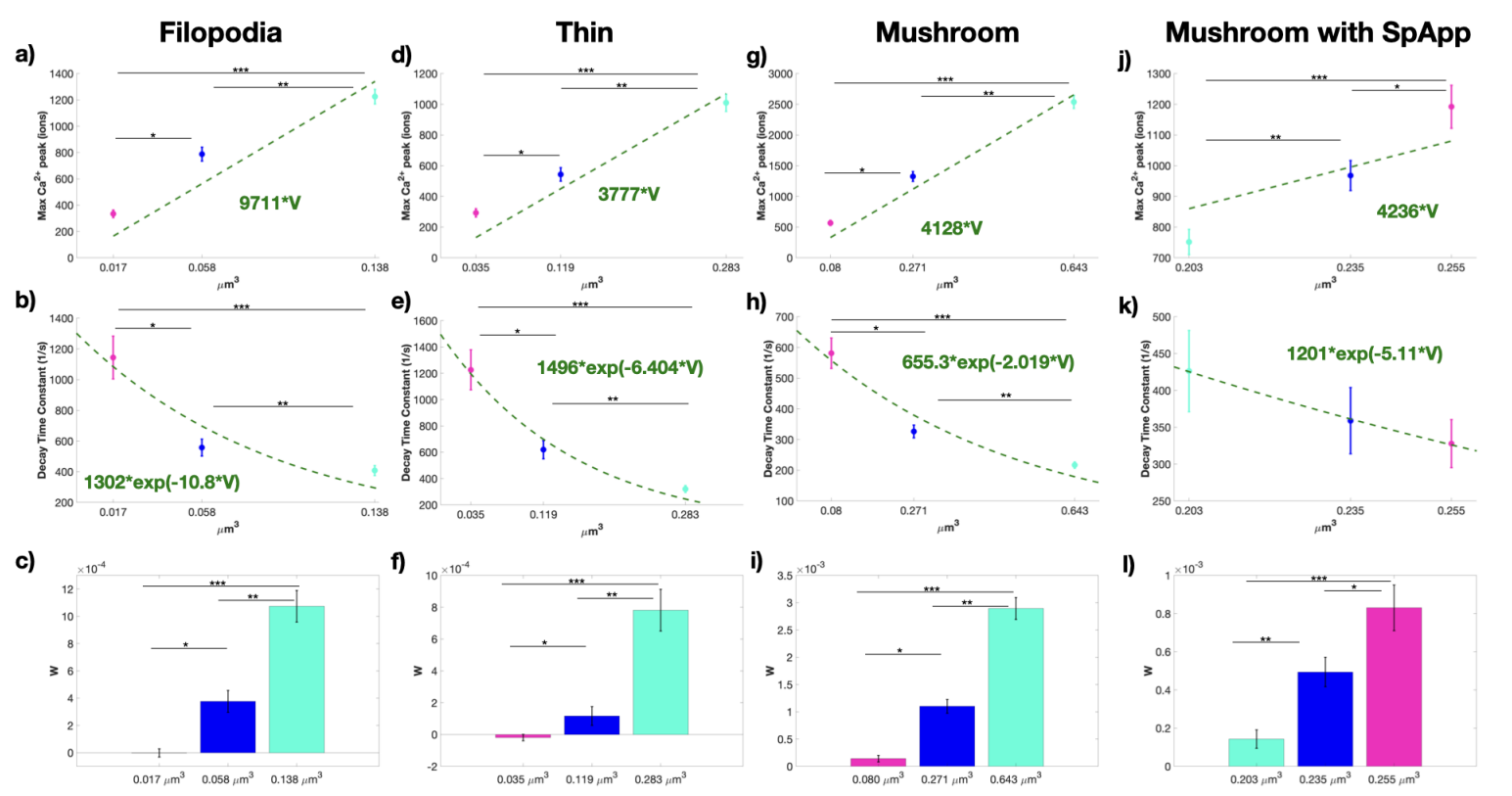

Figure S1: Trends across volume are similar to trends across volume-to-surface area ratio Peak calcium levels, decay time constant, and synaptic weight updates for size variations given as volumes for filopodia-shaped spines (a-c), thin spines (d-f), mushroom spines (g-i), and mushroom spines with spine apparatus (j-I). Peak calcium is fit with a line with a fixed zero intercept.

\section{S1.7 Supplemental movies}

\section{S1.7.1 Supplemental Movie S1}

Sample movie of idealized filopodia simulation. A single seed of an idealized filopodia simulation is shown for the whole time period from 0 to $35 \mathrm{~ms}$. The plasma membrane mesh is shown in blue and the $\mathrm{Ca}^{2+}$ ions are red.

\section{S1.7.2 Supplemental Movie S2}

Sample movie of idealized thin spine simulation. A single seed of an idealized thin spine simulation is shown for the whole time period from 0 to $35 \mathrm{~ms}$. The plasma membrane mesh is shown 
a)

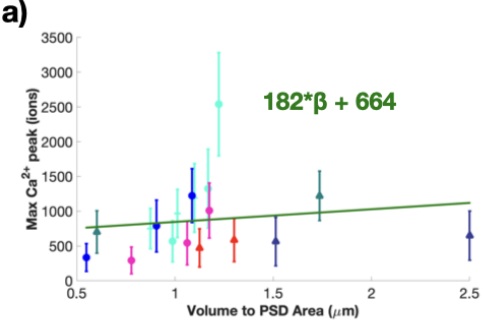

d)

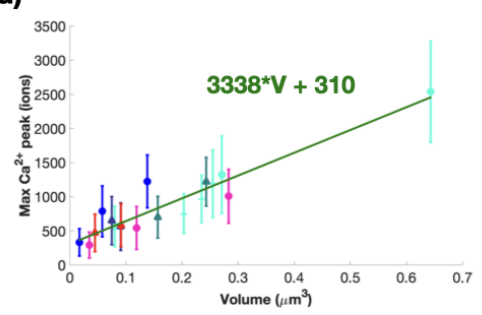

b)

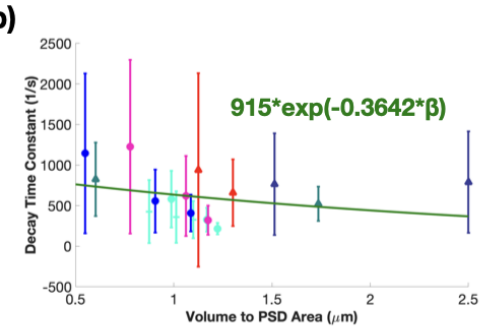

e)

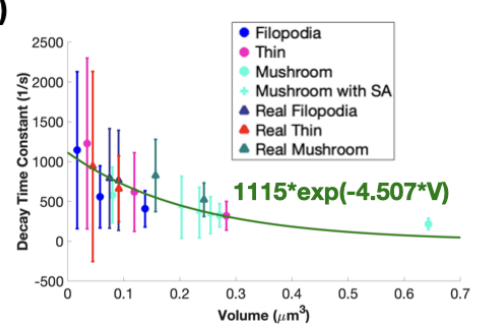

c)

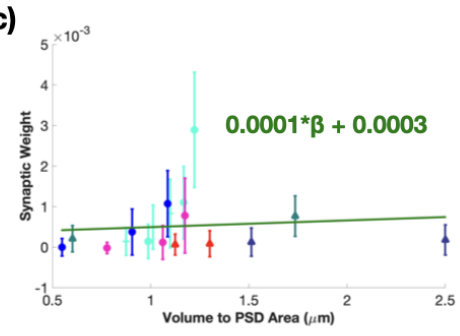

f)

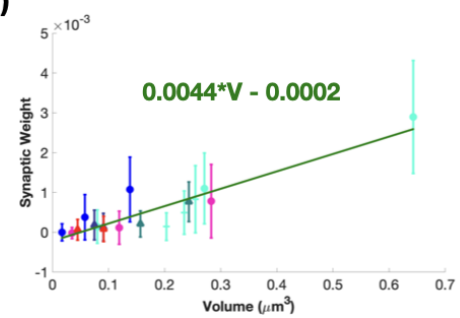

Figure S2: Trends across volume-to-PSD area ratio and across volume show different levels of significance a) All calcium peaks as mean and standard error $(n=50)$ across volume-to-PSD area ratio show no dependence. We fit the trend in peak values with a linear function against the volume-to-PSD area ratio; $r^{2}=0.0152$ for the linear fit. b) We fit the decay dynamics of each calcium transient with $c \cdot \exp (-k t)$ and report the decay time constant, $\mathrm{k}$, as a mean and standard error $(n=50)$ against volume-to-PSD area ratio. We fit the trend in decay time constants as a function of volume-to-PSD area ratio with an exponential $a \cdot \exp (-b \beta)$, where $\beta$ is the volume-to-PSD area ratio; $r^{2}=0.0091$ for the fit. c) Calculated synaptic weight change mean and standard error $(n=50)$ at the last time point for all idealized and realistic spines shows no dependence on volume-to-PSD area ratio. We fit the trend in synaptic weight change with a linear function against the volume-to-PSD area ratio; $r^{2}=0.0060$ for the linear fit. d) All calcium peaks as mean and standard error $(\mathrm{n}=50)$ across volume show a clear increasing trend. We fit the trend in peak values with a linear function against volume; $r^{2}=0.5666$ for the linear fit. e) We fit the decay dynamics of each calcium transient with $c \cdot \exp (-k t)$ and report the decay time constant, $k$, as a mean and standard error $(n=50)$ against volume. We fit the trend in decay time constants as a function of volume with an exponential $a \cdot \exp (-b V)$, where $\mathrm{V}$ is the volume; $r^{2}=0.1478$ for the fit. f) Calculated synaptic weight change mean and standard error $(n=50)$ at the last time point for all idealized and realistic spines shows an increasing trend. We fit the trend in synaptic weight change with a linear function against volume; $r^{2}$ $=0.4635$ for the linear fit. 

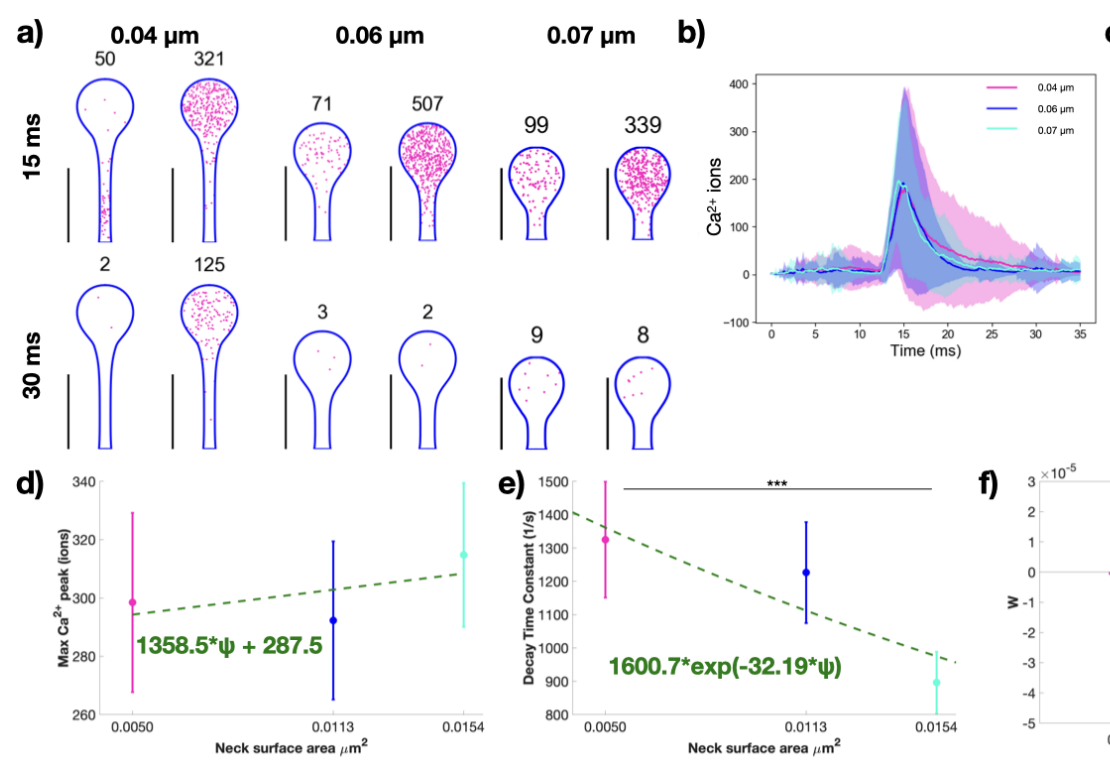

c)
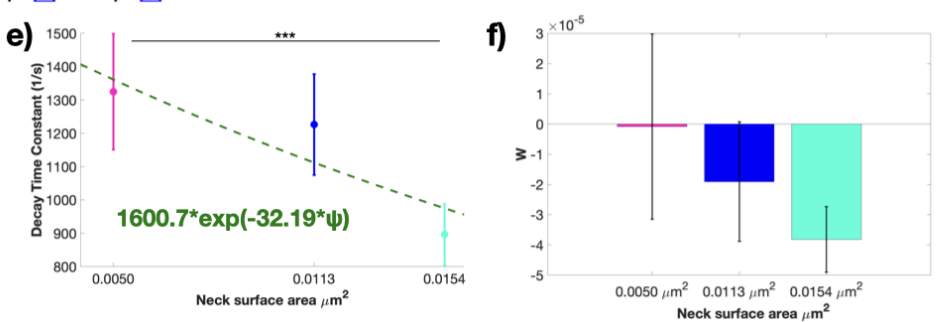

Figure S3: Effect of spine neck variation on synaptic plasticity in thin spines. a) Spatial plots at 15 and $30 \mathrm{~ms}$ for thin spines of the same volume with different neck geometries (neck radius of $0.04,0.06,0.07 \mu \mathrm{m}$ ). The number above each spine corresponds to the number of calcium ions present at that time point. Scale bar: $2 \mu \mathrm{m}$. Calcium ions over time (b) and variance (c) for all three thin spines with different neck cases. Shaded regions in (b) denote standard deviation. d) Peak calcium ion number for each thin spine with the mean and standard error $(n=50)$ show no statistically significant differences using a two-tailed $t$-test. We fit the trend in peak calcium as a linear function of spine neck base surface area; $r^{2}=0.0009$ for the linear fit. e) We fit the decay portion of each calcium transient with the exponential decay function $c \cdot \exp (-k t)$. The decay time constant mean and standard error $(\mathrm{n}=50), k$, only shows statistically significant differences between the thin and thick necks; $\mathrm{p}^{* * *}=0.0322$ from a two-tailed $t$-test. We fit the trend in decay time constants as a function of spine neck base surface area with an exponential $a \cdot \exp (-b \psi)$, where $\psi$ is the spine neck base surface area; $r^{2}=0.0256$ for the exponential fit. f) Calculated synaptic weight change at the last time point for all three thin spines shows no statistically significant difference due to neck size. 


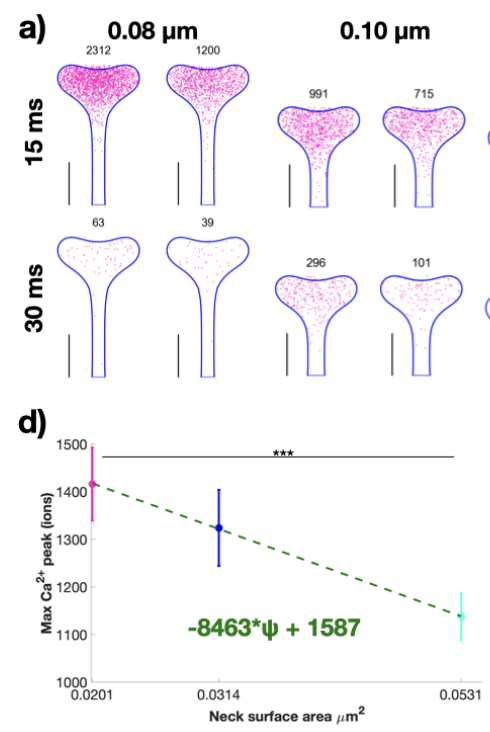

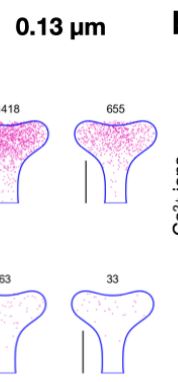

e)

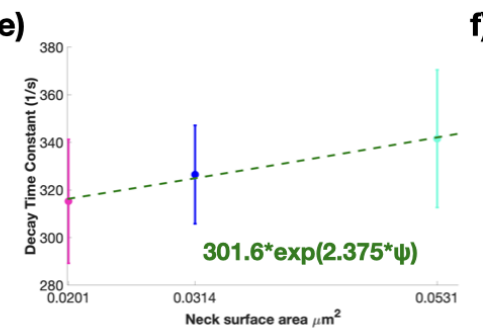

c)

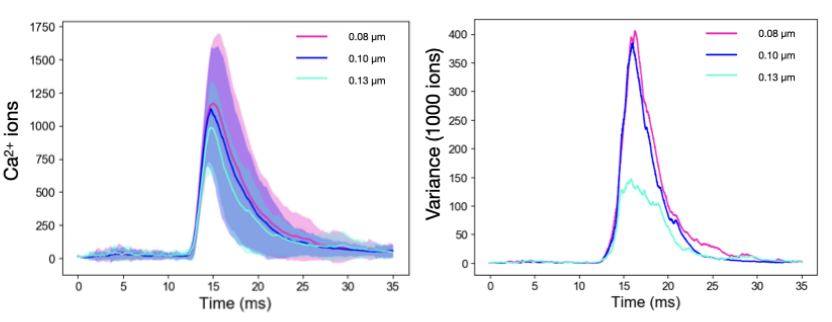

f)

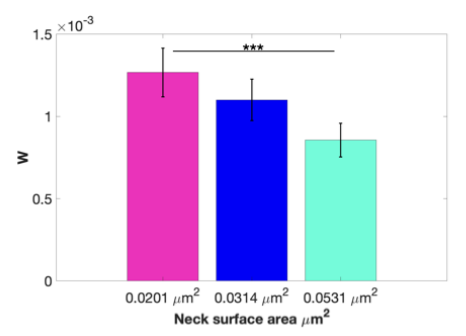

Figure S4: Effect of spine neck variation on synaptic plasticity in mushroom spines. a) Spatial plots at 15 and $30 \mathrm{~ms}$ for mushroom spines of the same volume with different neck geometries (neck radius of $0.08,0.10,0.13 \mu \mathrm{m}$ ). The number above each spine corresponds to the number of calcium ions present at that time point. Scale bar: $2 \mu \mathrm{m}$. Calcium ions over time (b) and variance (c) for all three mushroom spines with different neck cases. Shaded regions in (b) denote standard deviation. d) Peak calcium ion number for each mushroom spine with the mean and standard error $(n=50)$ show statistically significant differences between the thin and thick spines; $p^{* * *}=0.0029$ using a two-tailed $t$-test. We fit the trend in peak calcium as a linear function of spine neck base surface area; $r^{2}=0.0528$ for the linear fit. e) We fit the decay portion of each calcium transient with the exponential decay function $c \cdot \exp (-k t)$. The decay time constant mean and standard error $(\mathrm{n}=50), k$, shows no statistically significant differences from a two-tailed $t$-test. We fit the trend in decay time constants as a function of spine neck base surface area with an exponential $a \cdot \exp (-b \psi)$, where $\psi$ is the spine neck base surface area; $r^{2}=0.0036$ for the exponential fit. f) Calculated synaptic weight change at the last time point for all three mushroom spines only shows a statistically significant difference between the thin and thick spines, $\mathrm{p}^{* \star *}=0.0244$ from two-tailed $t$-test. 

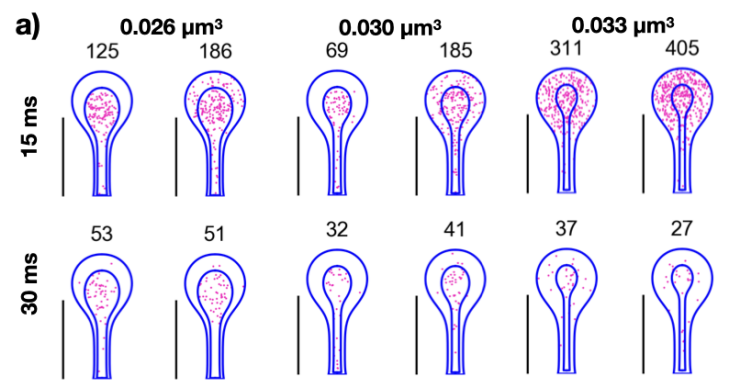

b)

c)
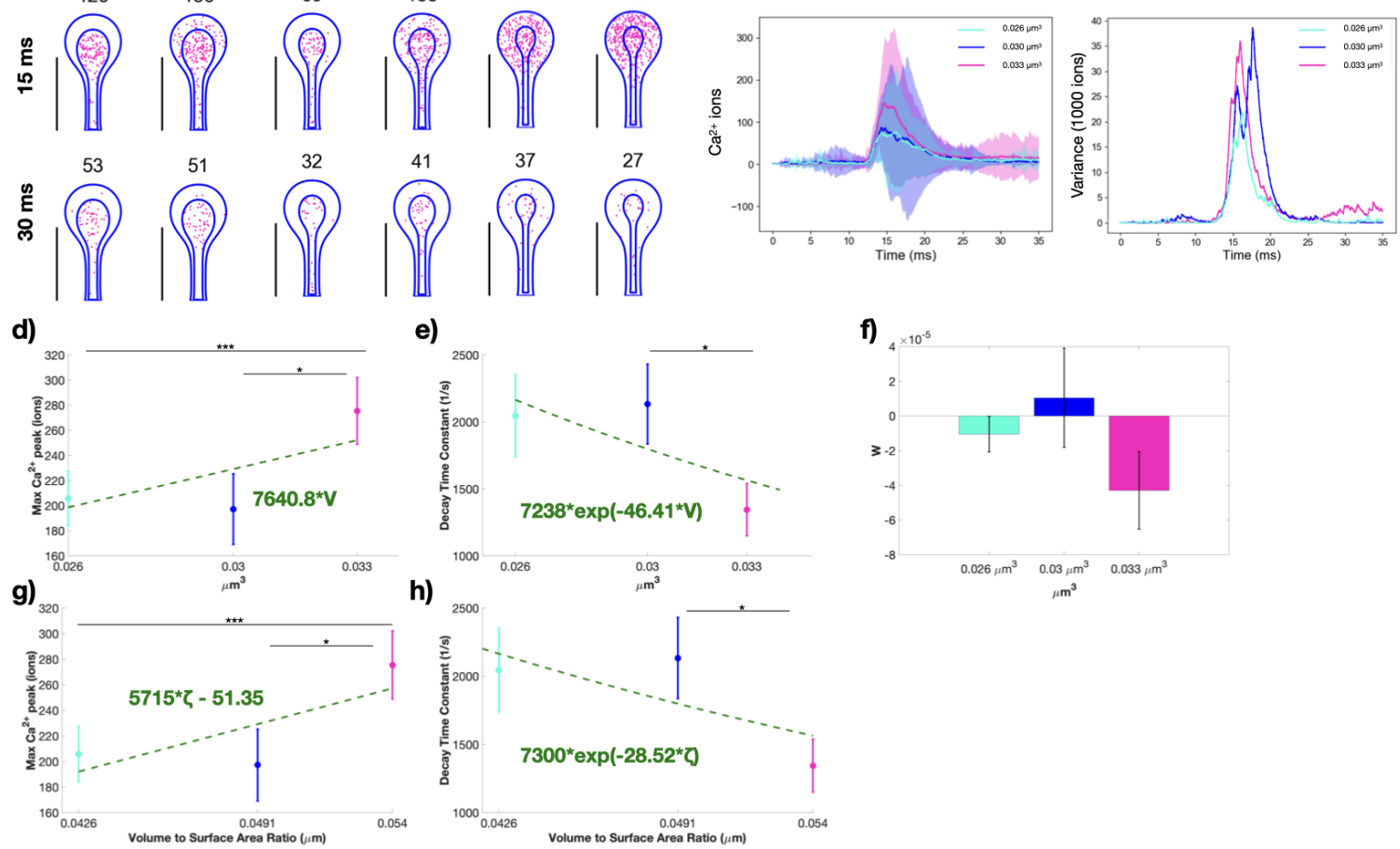

Figure S5: Spine apparatus size modulates synaptic weight change in thin spines. a) Spatial plots at 15 and 30 $\mathrm{ms}$ for thin spines with spine apparatus of different volumes (spine cytosolic volumes of $0.026,0.030,0.0 .033 \mu \mathrm{m}^{3}$ ). The numbers on top of the shape indicate the total number of calcium ions at that instant in both the spine apparatus and cytoplasm. Calcium ions over time as mean and standard deviation (b) and variance (c) for all three thin spines with different spine apparatus sizes. Shaded regions in (b) denote standard deviation. d) Peak calcium ion number for each thin spine with a spine apparatus, with the mean and standard error $(n=50)$, show statistically significant differences between two of the three paired cases; $\mathrm{p}^{*}=0.0461 ; \mathrm{p}^{* * *}=0.0453$ from two-tailed $t$-test. We fit the trend in peak values with a linear function against the cytoplasm volume; $r^{2}=0.0145$ for the linear fit. e) We fit the decay dynamics of each calcium transient with $c \cdot \exp (-k t)$ and report the decay time constant, $\mathrm{k}$, as a mean and standard error $(n=50)$. We find only find statistically significant differences between the second and third spines; $\mathrm{p}^{*}=0.0289$ from a two-tailed $t$-test. We fit the trend in decay time constants as a function of cytosolic volume with an exponential $a \cdot \exp (-b V)$, where $\mathrm{V}$ is the cytosolic volume; $r^{2}=0.0177$ for the fit. f) Calculated synaptic weight change at the last time point for all three thin spines shows no statistically significant difference due to spine apparatus size. We also plot peak calcium ion number and decay time constant against the cytosolic volume to surface area ratio, $\mathrm{g}$ and $\mathrm{h}$, respectively. $\mathrm{g}$ ) We fit the trend in peak values with a linear function against the volume-to-surface area ratio; $r^{2}=0.0214$ for the linear fit. $\mathrm{h}$ )We fit the trend in decay time constants as a function of volume-to-surface area ratio with an exponential $a \cdot \exp (-b \zeta)$, where $\zeta$ is the volume-to-surface area ratio; $r^{2}=0.0178$ for the fit. 
a)
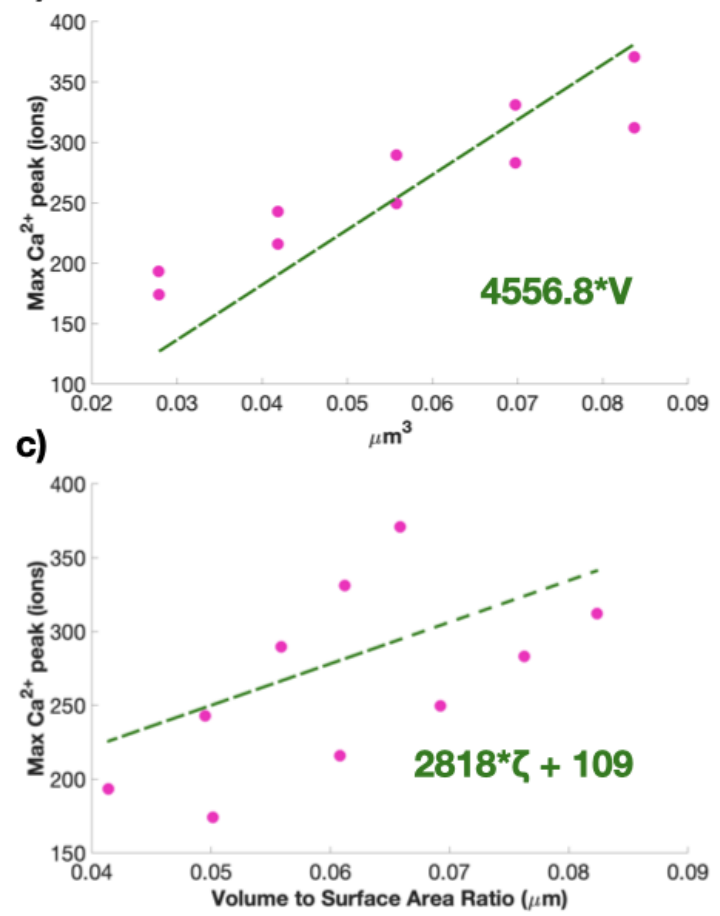

b)
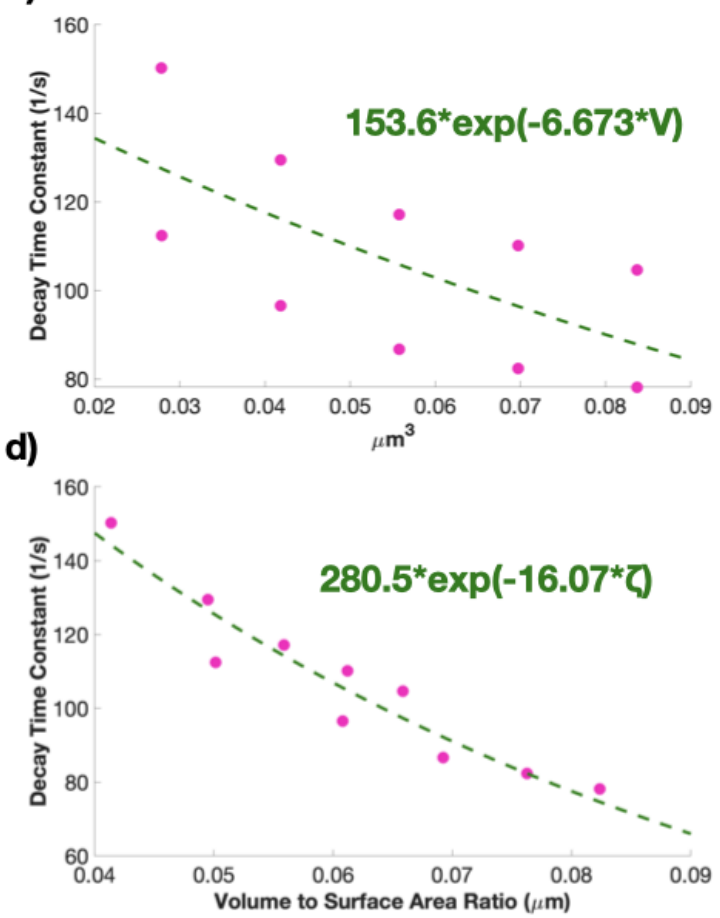

Figure S6: Previous calcium simulation results match the qualitative trends in these results. a) We fit the trend in peak values with a linear function against the cytoplasm volume; $r^{2}=0.8242$ for the linear fit. We fix the $y$ intercept at zero. b) We fit the decay dynamics of each calcium transient with $c \cdot \exp (-k t)$ and report the decay time constant, $\mathrm{k}$. We fit the trend in decay time constants as a function of cytosolic volume with an exponential $a \cdot \exp (-b V)$, where $\mathrm{V}$ is the cytosolic volume; $r^{2}=0.4283$ for the fit. c) We fit the trend in peak values with a linear function against the volume-to-surface area ratio; $r^{2}=0.8776$ for the linear fit. h) We fit the trend in decay time constants as a function of volume-to-surface area ratio with an exponential $a \cdot \exp (-b \zeta)$, where $\zeta$ is the volume-to-surface area ratio; $r^{2}=0.9054$ for the fit. 
a)

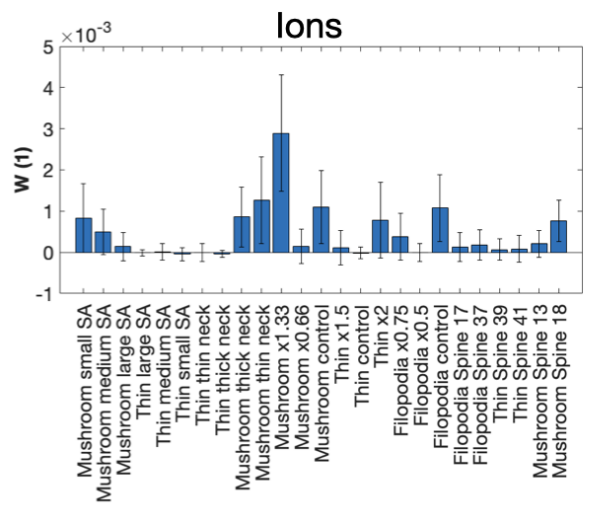

c)

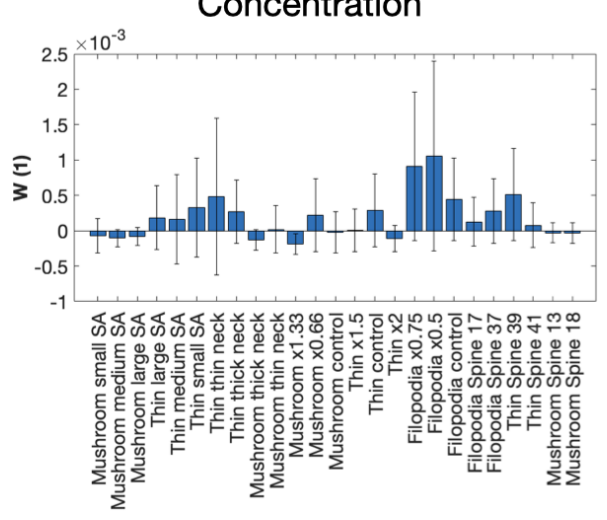

b)

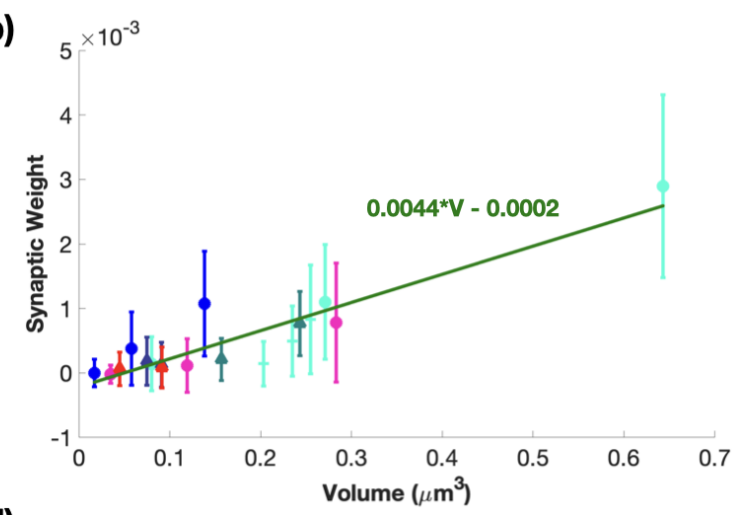

d)

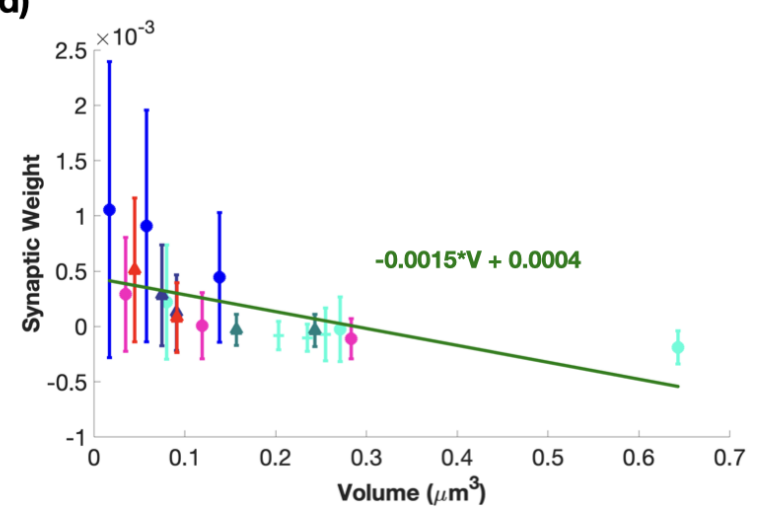

Figure S7: Synaptic weight updates when considering $\mathrm{Ca}^{2+}$ in terms of ions or concentration Synaptic weight updates for each stochastic idealized and real geometry simulation when synaptic weight calculations are in terms of ions (a-b) and concentration (c-d). We plot the synaptic weight changes against the spine volume for calculations using ions (b) and concentration (d). We fit the trends using a linear function of volume. We get $r^{2}=0.4635$ for the ion fit and $r^{2}=0.1229$ for the concentration fit. 

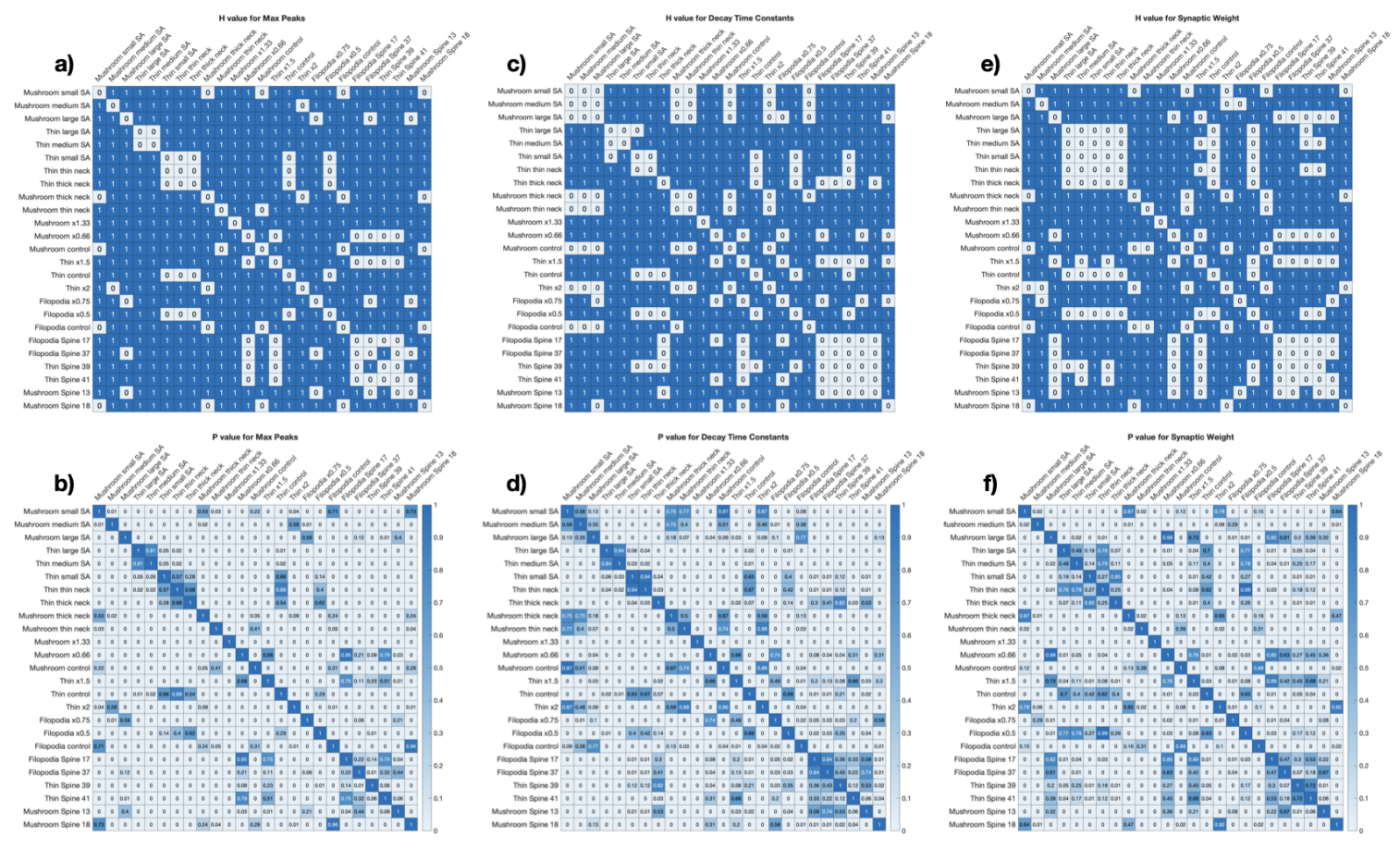

Figure S8: Two-tailed $t$-test comparison between all simulations We conduct two-tailed $t$-test between all simulations and display the $\mathrm{h}$ value and $\mathrm{p}$ value for max $\mathrm{Ca}^{2+}$ peaks $(\mathrm{a}-\mathrm{b})$, decay rate constant (c-d), and synaptic weight change (e-f). Displayed $p$ values are truncated at two decimal points.

in blue and the $\mathrm{Ca}^{2+}$ ions are red.

\section{S1.7.3 Supplemental Movie S3}

Sample movie of idealized mushroom spine simulation. A single seed of an idealized mushroom spine simulation is shown for the whole time period from 0 to $35 \mathrm{~ms}$. The plasma membrane mesh is shown in blue and the $\mathrm{Ca}^{2+}$ ions are red.

\section{S1.7.4 Supplemental Movie S4}

Sample movie of realistic mushroom spine 13 simulation. A single seed of a realistic mushroom spine 13 simulation is shown for the whole time period from 0 to $35 \mathrm{~ms}$. The plasma membrane mesh is shown in blue and the $\mathrm{Ca}^{2+}$ ions are red.

\section{S1.7.5 Supplemental Movie S5}

Sample movie of realistic filopodia 17 simulation. A single seed of a realistic filopodia 17 simulation is shown for the whole time period from 0 to $35 \mathrm{~ms}$. The plasma membrane mesh is shown in blue and the $\mathrm{Ca}^{2+}$ ions are red.

\section{S1.7.6 Supplemental Movie S6}

Sample movie of realistic mushroom spine 18 simulation. A single seed of a realistic mushroom spine 18 simulation is shown for the whole time period from 0 to $35 \mathrm{~ms}$. The plasma mem- 
brane mesh is shown in blue and the $\mathrm{Ca}^{2+}$ ions are red.

\section{S1.7.7 Supplemental Movie S7}

Sample movie of realistic filopodia 37 simulation. A single seed of a realistic filopodia 37 simulation is shown for the whole time period from 0 to $35 \mathrm{~ms}$. The plasma membrane mesh is shown in blue and the $\mathrm{Ca}^{2+}$ ions are red.

\section{S1.7.8 Supplemental Movie S8}

Sample movie of realistic thin spine 39 simulation. A single seed of a realistic thin spine 39 simulation is shown for the whole time period from 0 to $35 \mathrm{~ms}$. The plasma membrane mesh is shown in blue and the $\mathrm{Ca}^{2+}$ ions are red.

\section{S1.7.9 Supplemental Movie S9}

Sample movie of realistic thin spine 41 simulation. A single seed of a realistic thin spine 41 simulation is shown for the whole time period from 0 to $35 \mathrm{~ms}$. The plasma membrane mesh is shown in blue and the $\mathrm{Ca}^{2+}$ ions are red. 


\section{References}

1. J. Nishiyama, R. Yasuda, Neuron 87, 63-75 (2015).

2. H. Murakoshi, R. Yasuda, Trends in neurosciences 35, 135-143 (2012).

3. R. Yuste, W. Denk, Nature 375, 682 (1995).

4. R. Yuste, A. Majewska, K. Holthoff, Nature neuroscience 3, 653 (2000).

5. G. J. Augustine, F. Santamaria, K. Tanaka, Neuron 40, 331-346 (2003).

6. C. E. Jahr, C. F. Stevens, Proceedings of the National Academy of Sciences 90, 1157311577 (1993).

7. H. Z. Shouval, M. F. Bear, L. N. Cooper, Proceedings of the National Academy of Sciences 99, 10831-10836 (2002).

8. M. Graupner, N. Brunel, Proceedings of the National Academy of Sciences 109, 3991-3996 (2012).

9. C. O’Donnell, M. F. Nolan, M. C. van Rossum, Journal of Neuroscience 31, 16142-16156 (2011).

10. R. C. Malenka, J. A. Kauer, R. S. Zucker, R. A. Nicoll, Science 242, 81-84 (1988).

11. J. A. Cummings, R. M. Mulkey, R. A. Nicoll, R. C. Malenka, Neuron 16, 825-833 (1996).

12. L. C. Yeung, H. Z. Shouval, B. S. Blais, L. N. Cooper, Proceedings of the National Academy of Sciences 101, 14943-14948 (2004).

13. K. F. Lee, C. Soares, J.-C. Béı $\square$ que, Neural plasticity 2012 (2012).

14. J. Lisman, Philosophical Transactions of the Royal Society B: Biological Sciences 372, 20160260 (2017).

15. B. A. Earnshaw, P. C. Bressloff, Journal of Neuroscience 26, 12362-12373 (2006).

16. J. Jędrzejewska-Szmek, S. Damodaran, D. B. Dorman, K. T. Blackwell, European Journal of Neuroscience 45, 1044-1056 (2017).

17. J. N. Bourne, K. M. Harris, Annu. Rev. Neurosci. 31, 47-67 (2008).

18. R. Calizo et al., Scientific reports 10, 1-17 (2020).

19. S. R. Neves et al., Cell 133, 666-680 (2008).

20. P. Rangamani et al., Cell 154, 1356-1369 (2013).

21. L. Héja, Z. Szabó, M. Péter, J. Kardos, Frontiers in Cellular Neuroscience 15, 14 (2021).

22. M. Bell, T. Bartol, T. Sejnowski, P. Rangamani, Journal of General Physiology 151, 10171034 (2019).

23. A. Cugno, T. M. Bartol, T. J. Sejnowski, R. Iyengar, P. Rangamani, Scientific reports 9, 1-18 (2019).

24. T. M. Bartol et al., Frontiers in synaptic neuroscience 7, 17 (2015).

25. N. Volfovsky, H. Parnas, M. Segal, E. Korkotian, Journal of neurophysiology 82, 450-462 (1999).

26. D. Holcman, Z. Schuss, E. Korkotian, Biophysical journal 87, 81-91 (2004).

27. M. J. Higley, B. L. Sabatini, Neuron 59, 902-913 (2008).

28. H. Anwar, I. Hepburn, H. Nedelescu, W. Chen, E. De Schutter, Journal of Neuroscience 33, 15848-15867 (2013).

29. J. T. Dudman, M. F. Nolan, PLoS Comput Biol 5, e1000290 (2009).

30. A. A. Faisal, J. A. White, S. B. Laughlin, Current Biology 15, 1143-1149 (2005).

31. R. C. Cannon, C. O’Donnell, M. F. Nolan, PLoS Comput Biol 6, e1000886 (2010).

32. C. O'Donnell, M. F. Nolan, in The Computing Dendrite (Springer, 2014), pp. 397-414. 
33. J. R. Stiles, T. M. Bartol, et al., Computational neuroscience: realistic modeling for experimentalists, 87-127 (2001).

34. J. R. Stiles, D. Van Helden, T. M. Bartol, E. E. Salpeter, M. M. Salpeter, Proceedings of the National Academy of Sciences of the United States of America, 5747-5752 (1996).

35. R. A. Kerr et al., SIAM journal on scientific computing 30, 3126-3149 (2008).

36. H. Alimohamadi, M. Bell, S. Halpain, P. Rangamani, bioRxiv (2020).

37. A. S. Ozcan, Frontiers in synaptic neuroscience 9, 12 (2017).

38. P. García-López, V. García-Marín, M. Freire, Neural plasticity 2010 (2010).

39. N. Ofer, D. R. Berger, N. Kasthuri, J. W. Lichtman, R. Yuste, bioRxiv, DOI 10.1101/2021. 02.18 .431725 (2021).

40. R. Yuste, Dendritic spines (MIT press, 2010).

41. M. Jasinska et al., PloS one 14, e0225394 (2019).

42. M. A. Chirillo, M. S. Waters, L. F. Lindsey, J. N. Bourne, K. M. Harris, Scientific reports $\mathbf{9}$, 1-14 (2019).

43. J. Spacek, K. M. Harris, Journal of Neuroscience 17, 190-203 (1997).

44. A. Perez-Alvarez et al., Nature communications 11, 1-10 (2020).

45. B. L. Sabatini, T. G. Oertner, K. Svoboda, Neuron 33, 439-452 (2002).

46. Y. Wu et al., Proceedings of the National Academy of Sciences 114, E4859-E4867 (2017).

47. C. T. Lee et al., PLOS Comp Bio (2020).

48. J. H. Kotaleski, K. T. Blackwell, Nature Reviews Neuroscience 11, 239-251 (2010).

49. R. Yasuda, Biophysical journal 113, 2152-2159 (2017).

50. R. Yuste, T. Bonhoeffer, Nature Reviews Neuroscience 5, 24-34 (2004).

51. I. S. Stein, K. Zito, The Neuroscientist 25, 27-47 (2019).

52. D. A. Ruhl et al., Nature communications 10, 1-14 (2019).

53. C. Lohmann, T. Bonhoeffer, Neuron 59, 253-260 (2008).

54. K. E. Sorra, K. M. Harris, Hippocampus 10, 501-511 (2000).

55. G. Mahajan, S. Nadkarni, The Journal of physiology 597, 3473-3502 (2019).

56. R. L. Wasserstein, A. L. Schirm, N. A. Lazar, Moving to a world beyond " $p<0.05$ ", 2019.

57. D. Ohadi, P. Rangamani, Biophysical journal 117, 1981-1994 (2019).

58. T. Mäki-Marttunen, N. lannella, A. G. Edwards, G. T. Einevoll, K. T. Blackwell, Elife 9, e55714 (2020).

59. U. S. Bhalla, Biophysical Journal 87, 733-744 (2004).

60. D. Ohadi et al., Biophysical journal 117, 1963-1980 (2019).

61. C. Miermans, R. Kusters, C. Hoogenraad, C. Storm, PloS one 12, e0170113 (2017).

62. K. Basnayake et al., PLoS biology 17, e2006202 (2019).

63. M. K. Bell, P. Rangamani, Current Opinion in Systems Biology (2021).

64. C. T. Lee, M. Akamatsu, P. Rangamani, Current Opinion in Cell Biology 71, 38-45 (2021).

65. A. Hayer, U. S. Bhalla, PLoS Comput Biol 1, e20 (2005).

66. M. Ordyan, T. Bartol, M. Kennedy, P. Rangamani, T. Sejnowski, PLoS computational biology 16, e1008015 (2020).

67. M. Bonilla-Quintana, F. Wörgötter, C. Tetzlaff, M. Fauth, Frontiers in synaptic neuroscience 12, 9 (2020).

68. P. Rangamani, G. Y. Xiong, R. lyengar, Progress in molecular biology and translational science 123, 143-167 (2014). 
69. L. N. Cornelisse, R. A. van Elburg, R. M. Meredith, R. Yuste, H. D. Mansvelder, PloS one 2, e1073 (2007).

70. R. Robinson, R. Stokes, Electrolyte Solutions, Butterworths Scientific Publications, 1959.

71. B. Schwaller, Cold Spring Harbor perspectives in biology, a004051 (2010).

72. M. Vargas-Caballero, H. P. Robinson, Journal of Neuroscience 24, 6171-6180 (2004).

73. P. Jonas, G. Major, B. Sakmann, Journal of Physiology 472, 615-663 (1993).

74. T. Griffith, K. Tsaneva-Atanasova, J. R. Mellor, PLoS computational biology 12, e1004949 (2016).

75. J. Schoberl, Comput Visual Sci 1, 41-52 (1997).

76. M. Borczyk, M. A. Sliwinska, A. Caly, T. Bernas, K. Radwanska, Scientific Reports 9 (2019).

77. C. T. Lee et al., Biophysical Journal 118, 1003-1008, issn: 0006-3495, DOI $10.1016 / \mathrm{j}$. bpj . 2019 . 11 . 3400, (2020; http : / / www . sciencedirect . com/science/article/pii / S0006349520300357) (Mar. 10, 2020). 